November, 1996

IASSNS-HEP-96/121

hep-th/9611230

\title{
Type IIB Superstrings, BPS Monopoles, And Three-Dimensional Gauge Dynamics
}

\author{
Amihay Hanany and Edward Witten * \\ hanany; witten@ias.edu \\ School of Natural Sciences \\ Institute for Advanced Study \\ Olden Lane, Princeton, NJ 08540, USA
}

\begin{abstract}
We propose an explanation via string theory of the correspondence between the Coulomb branch of certain three-dimensional supersymmetric gauge theories and certain moduli spaces of magnetic monopoles. The same construction also gives an explanation, via $S L(2, \mathbf{Z})$ duality of Type IIB superstrings, of the recently discovered "mirror symmetry" in three dimensions. New phase transitions in three dimensions as well as new infrared fixed points and even new coupling constants not present in the known Lagrangians are predicted from the string theory construction. An important role in the construction is played by a novel aspect of brane dynamics in which a third brane is created when two branes cross.
\end{abstract}

${ }^{*}$ Research supported in part by NSF Grant PHY-9513835. 


\section{Introduction}

Recently, there has been much investigation of the dynamics of three-dimensional supersymmetric gauge theories with $N=4$ supersymmetry. Two of the most remarkable discoveries are as follows.

1. In some cases, the Coulomb branch of vacua is isomorphic, as a hyper-Kahler manifold, to a moduli space of three-dimensional monopole solutions in a different gauge theory. For instance, the three-dimensional theory with $S U(k)$ gauge theory and no hypermultiplets has for its Coulomb branch the moduli space of $k$-monopole configurations of an $S U(2)$ gauge theory in $3+1$ dimensions. (This was shown in [1] for $k=2$; the generalization to higher $k$ was proposed in [2].)

2. In some cases, there is a "mirror symmetry" between two models in which the Coulomb branch of one is the Higgs branch of the other and vice-versa [3].

The purpose of this paper is to give an explanation of these two phenomena by embedding them in a suitable string theory context. More specifically, we will describe a simple configuration of threebranes and fivebranes in ten-dimensional Type IIB superstring theory from whose basic properties the phenomena mentioned in the last paragraph can be deduced - at least for a large class of models. In the process, we will encounter a number of unusual phenomena, including field theories that can be generalized to include parameters not seen in the classical Lagrangian as well as things that are by now more familiar, such as exotic phase transitions and infrared fixed points. Also, in the course of our investigation, we will have to understand a new feature of brane dynamics in which a third brane is created when two branes cross.

\section{$2 \quad$ A Type IIB configuration}

In this paper, we will study certain supersymmetric configurations of fivebranes and threebranes in Type IIB superstring theory. In detail these will be as follows.

We work in ten-dimensional Minkowski space with time coordinate $x^{0}$ and space coordinates $x^{1}, \ldots, x^{9}$. Let $Q_{L}$ and $Q_{R}$ be the supercharges generated by left- and rightmoving world-sheet degrees of freedom. The Type IIB theory is chiral; the supercharges obey $\bar{\Gamma} Q_{L}=Q_{L}, \bar{\Gamma} Q_{R}=Q_{R}$, with $\bar{\Gamma}=\Gamma_{0} \Gamma_{1} \cdots \Gamma_{9}$.

Consider first a Dirichlet fivebrane located at $x^{6}=x^{7}=x^{8}=x^{9}=0$. It is invariant under linear combinations $\epsilon^{L} Q_{L}+\epsilon^{R} Q_{R}$ of supersymmetries with $\epsilon^{L}$, $\epsilon^{R}$ being spinors 
such that

$$
\epsilon_{L}=\Gamma_{0} \Gamma_{1} \Gamma_{2} \Gamma_{3} \Gamma_{4} \Gamma_{5} \epsilon_{R}
$$

Of course, the unbroken supersymmetry is the same if the fivebrane is located at any constant values of $x^{6} \ldots, x^{9}$, not necessarily zero.

Now consider an NS fivebrane located at definite values of $x^{6} \ldots x^{9}$. It is likewise invariant under half of the supersymmetries, namely those with

$$
\epsilon_{L}=\Gamma_{0} \Gamma_{1} \cdots \Gamma_{5} \epsilon_{L}, \quad \epsilon_{R}=-\Gamma_{0} \Gamma_{1} \cdots \Gamma_{5} \epsilon_{R}
$$

One way to obtain this result is to apply an $S L(2, \mathbf{Z})$ duality transformation which converts the Dirichlet fivebrane to an NS fivebrane and maps (2.1) to (2.2). Another approach is to directly study the conformal field theory of the fivebrane, which is known [4-6] not exactly but well enough to identify the unbroken supersymmetries. In this description it is obvious that the conformal field theory has no mixing between left- and right-moving modes, which is the reason that $\epsilon_{L}$ and $\epsilon_{R}$ obey independent conditions in (2.2). This together with Lorentz invariance determines the form of (2.2) up to signs, and with one choice of what we mean by an NS fivebrane (as opposed to an anti-fivebrane) the signs are as in (2.2).

Now we want to consider a supersymmetric configuration with both NS and Dirichlet fivebranes. A comparison of the above formulas shows that if both types of fivebrane are present at definite values of $x^{6}, \ldots, x^{9}$, then no supersymmetry at all is preserved. Suppose, however, that we have an NS fivebrane at definite values of $x^{6}, \ldots, x^{9}$ and a Dirichlet fivebrane at definite values of $x^{3}, x^{4}, x^{5}, x^{6}$. Then (2.2) is unchanged, but (2.1) becomes

$$
\epsilon_{L}=\Gamma_{0} \Gamma_{1} \Gamma_{2} \Gamma_{7} \Gamma_{8} \Gamma_{9} \epsilon_{R} .
$$

Comparing (2.2) and (2.3), we find that this sort of configuration preserves one quarter of the supersymmetries.

There is one further kind of brane that can be introduced without any further breaking of supersymmetry. If we combine (2.2) and (2.3), we deduce that

$$
\epsilon_{L}=\Gamma_{0} \Gamma_{1} \Gamma_{2} \Gamma_{6} \epsilon_{R} .
$$

Supersymmetries generated by parameters obeying this equation are precisely those that are unbroken in the presence of a Dirichlet threebrane whose world-volume spans the $x^{0}, x^{1}, x^{2}$, and $x^{6}$ directions and is appropriately oriented (that is, it is a threebrane rather than an antithreebrane, which would give a minus sign in 2.4). So we can add such threebranes without any additional supersymmetry breaking. 
The presence of all of these branes breaks the Lorentz group $S O(1,9)$ to $S O(1,2) \times$ $S O(3) \times S O(3)$, where $S O(1,2)$ acts on $x^{0}, x^{1}, x^{2}$, one $S O(3)$ acts on $\vec{m}=\left(x^{3}, x^{4}, x^{5}\right)$, and one $S O(3)$ acts on $\vec{w}=\left(x^{7}, x^{8}, x^{9}\right)$. We will call the $S O(3)$ 's respectively $S O(3)_{V}$ and $S O(3)_{H}$; their double covers $S U(2)_{V}$ and $S U(2)_{H}$ will act, respectively, as symmetries of the Coulomb and Higgs branches.

From what we have said so far, we will be considering NS fivebranes at definite values of $x^{6}$ and $\vec{w}$, and D fivebranes at definite values of $x^{6}$ and $\vec{m}$. We will write $t_{i}$ and $\vec{w}_{i}$ for the values of $x^{6}$ and $\vec{w}$ of the $i^{t h}$ NS fivebrane, and $z_{j}$ and $\vec{m}_{j}$ for the values of $x^{6}$ and $\vec{m}$ of the $j^{\text {th }}$ D fivebrane.

The threebranes that we consider will not be infinite threebranes; we will consider threebranes ending on fivebranes. (Starting with the fact that elementary strings can end on Type IIB threebranes and applying various perturbative and nonperturbative dualities, one can deduce $[7,8]$ that threebranes can end on arbitrary fivebranes.) Three kinds of threebrane will appear: those with both ends on an NS fivebrane, those with both ends on a D fivebrane, and those with one end on one kind of fivebrane and the other on the other kind of fivebrane.

To preserve supersymmetry, a threebrane must have its world-volume precisely in the $x^{0}, x^{1}, x^{2}, x^{6}$ directions, so a threebrane can connect two given fivebranes in a supersymmetric configuration only if the transverse positions of the fivebranes obey certain constraints. To be precise, two NS fivebranes $i$ and $i^{\prime}$ can be connected by a threebrane if and only if $\vec{w}_{i}=\vec{w}_{i^{\prime}}$, and a threebrane connecting them has an arbitrary value of $\vec{m}$ (since the NS fivebrane world-volumes range over all values of $\vec{m}$ ). We will write $\vec{x}_{\alpha}$ for the value of $\vec{m}$ of the $\alpha^{\text {th }}$ threebrane. Likewise, two D fivebranes $j$ and $j^{\prime}$ can be connected by a threebrane if and only if $\vec{m}_{j}=\vec{m}_{j^{\prime}}$, and a threebrane connecting them has an arbitrary value of $\vec{w}$. We will write $\vec{y}_{\alpha}$ for the value of $\vec{w}$ of the $\alpha^{\text {th }}$ threebrane. Finally, any NS fivebrane can be connected to any D fivebrane by a threebrane, since the NS fivebrane spans all $\vec{m}$ and the D fivebrane spans all $\vec{w}$. But there are no moduli in the position of a threebrane connecting an NS fivebrane to a D fivebrane; the $\vec{w}$ value of such a threebrane is that of the NS fivebrane, and its $\vec{m}$ value is that of the $\mathrm{D}$ fivebrane.

A virtue of this setup, which will ultimately lead to our explanation of mirror symmetry, is that there is a symmetry between the two kinds of fivebrane. In fact, an $S L(2, \mathbf{Z})$ transformation by the matrix

$$
S=\left(\begin{array}{cc}
0 & 1 \\
-1 & 0
\end{array}\right)
$$

exchanges the two kinds of fivebrane. Let $\mathrm{R}$ be a rotation that maps $x^{j}$ to $x^{j+4}$ and $x^{j+4}$ 
to $-x^{j}$ for $j=3,4,5$ and leaves other coordinates invariant. The combined operation RS maps the class of configurations we are considering to itself, while exchanging the two kinds of fivebrane. We will call this mirror symmetry, justifying the name in due course.

\section{Field theory on the D3 brane}

Our general point of view in studying this problem is that because the fivebranes are infinite in two directions not shared by the threebranes, we can think of the fivebranes as being much heavier than the threebranes. Therefore, we think of the fivebrane parameters as being fixed, and we study the quantum dynamics of the threebrane motion, by analogy with many other investigations of field theory on branes, such as $[9,10]$. The parameters specifying the fivebrane positions $-z, t, \vec{w}$, and $\vec{m}$ - will be interpreted as coupling constants in an effective quantum field theory on the threebrane world volume. The positions of the D3 branes are dynamical moduli which parametrize the vacua for the field theory.

Since each threebrane we consider has only three infinite directions $x^{0}, x^{1}$, and $x^{2}$ (and spans only a finite extent in $x^{6}$ ), the threebrane field theory is macroscopically $2+1$-dimensional. This is rather like Kaluza-Klein theory where one compactifies on a circle and thereby reduces to a theory with a smaller number of macroscopic spacetime dimensions. The number of supercharges preserved by the configuration is 8 so we are dealing with $N=4$ supersymmetry in three dimensions. $N=4$ supersymmetry in three dimensions has potentially an $S O(4)=S U(2)_{V} \times S U(2)_{H}$ R-symmetry. For the class of models we are considering, those symmetries are actually present: they can be identified with the groups $S U(2)_{V}$ and $S U(2)_{H}$ already introduced in the last section. Incidentally, while there are many ways to embed a low energy field theory in string theory, in most instances the $R$ symmetries - which are very important in the field theory dynamics are only approximate symmetries of the string theory. The fact that in the approach considered here the $R$ symmetries are visible directly in string theory is one reason for the power of the construction.

To determine which $2+1$-dimensional field theories we obtain by this construction, we begin by considering a single infinite threebrane. We will write $N$ for the number of supersymmetries counted in three dimensions and $\mathcal{N}$ for the number counted in four dimensions. The world-volume theory on an infinite threebrane is a four-dimensional theory with twice as much supersymmetry as the models we will actually be studying in this paper; it has $\mathcal{N}=4$ supersymmetry in the four-dimensional sense or $N=8$ from

a three-dimensional point of view. The field theory on the infinite threebrane is a $U(1)$ 
gauge theory with a supermultiplet that is irreducible under $N=8$ (or $\mathcal{N}=4)$. But under the $N=4$ subalgebra that will actually be a symmetry of the models we will consider (in which the threebranes end on fivebranes), the ireducible $N=8$ multiplet decomposes as the sum of a vector multiplet and a hypermultiplet.

When a threebrane ends on a fivebrane, there are boundary conditions that set to zero half of the massless fields on the threebrane world-volume. It is important to know which half. Supersymmetry alone would allow boundary conditions in which either the vector multiplet or the hypermultiplet vanishes on the boundary (that is, obeys Dirichlet boundary conditions), while the other is free (obeys Neumann boundary conditions). In more detail, the possible boundary conditions are as follows.

1. A scalar field in $3+1$ dimensions can obey either Dirichlet boundary conditions, in which the scalar vanishes on the boundary, or Neumann boundary conditions, in which the boundary values are unconstrained but the normal derivative vanishes on the boundary.

2. A vector field in $3+1$ dimensions may obey either Dirichlet boundary conditions, in which the components of $F_{\mu \nu}$ with $\mu$ and $\nu$ tangent to the boundary vanish, or Neumann boundary conditions, in which the components in which one index is tangent to the boundary vanish. Note that a vector field $A$ in $3+1$ dimensions reduces to two fields in $2+1$ dimensions. If (as in the above conventions), we call the normal coordinate to the boundary $x^{6}$, and the other coordinates $x^{\mu}(\mu=0,1,2)$, the two $2+1$-dimensional fields are a scalar $b$ such that $\partial_{\mu} b=F_{\mu 6}$ and a $2+1$-dimensional $U(1)$ gauge field $a$ such that $a_{\mu}$ for $\mu=0,1,2$ is the $x^{6}$-independent component of $A_{\mu}$. (In the free abelian theory, one can make a duality transformation to turn $a_{\mu}$ into another scalar.) Neumann boundary conditions on $A$ set the scalar $b$ to zero in the effective $2+1$-dimensional theory, and Dirichlet boundary conditions set the vector $a$ to zero. Note that any transformation that acts as electric-magnetic duality on $A$ exchanges the components of $F_{\mu \nu}$ with both indices tangent to the boundary with components with one normal index, and so exchanges $b$ with the scalar dual to $a_{\mu}$ and exchanges the two types of boundary condition on $A$.

The potential massless modes in the effective $2+1$ dimensional theory on a threebrane are the fluctuations in the transverse position $\vec{x}$ and $\vec{y}$ (introduced in the last section) plus the scalar $b$ and the vector $a_{\mu}$. Under the $N=4$ algebra described in equations (2.1) - (2.4), $\vec{x}$ and $a_{\mu}$ form the bosonic part of a vector multiplet, while $\vec{y}$ and $b$ form the bosonic part of a hypermultiplet. Therefore, when a threebrane ends on one of the 
fivebranes introduced above, the boundary conditions are such that the bosonic modes not set to zero on the boundary are either $\vec{x}$ and $a_{\mu}$ or $\vec{y}$ and $b$.

At this point it is easy to deduce what happens. When a threebrane ends on an NS fivebrane, $\vec{x}$ is not set to zero because it is free to fluctuate in the case of a threebrane suspended between two fivebranes, as described in the next to last paragraph of the previous section. So for a threebrane ending on an NS fivebrane there are boundary conditions which set to zero $\vec{y}$ and $b$ and leave $\vec{x}$ and $a_{\mu}$ as massless modes. Conversely, $\vec{y}$ is not set to zero when a threebrane ends on a D fivebrane, as it is free to fluctuate for a threebrane suspended between two D fivebranes. So the boundary conditions for a threebrane ending on a D fivebrane set to zero $\vec{x}$ and $a_{\mu}$ and leave $\vec{y}$ and $b$ as massless modes.

Note that these statements are compatible with the mirror symmetry transformation introduced at the end of the last section. This exchanges the two kinds of fivebrane, exchanges $\vec{x}$ with $\vec{y}$, and (because it acts as electric-magnetic duality on the threebrane theory $[11,12])$ exchanges $b$ with the scalar dual to $a_{\mu}$.

Now the effective $2+1$ dimensional theory on a threebrane can be identified.

1. If a threebrane has both ends on a NS fivebrane, the effective $2+1$-dimensional theory is that of a $U(1)$ vector multiplet. More generally, given $n_{v}$ parallel threebranes suspended between the same two NS fivebranes, we get $n_{v}$ vector multiplets; the $U(1)^{n_{v}}$ gauge symmetry is at the "classical level" enhanced to $U\left(n_{v}\right)$ via Chan-Paton factors when the parallel threebranes become coincident. What really happens in the regime in which they are nearby is the problem of understanding the dynamics of the Coulomb branch of the $U\left(n_{v}\right)$ quantum gauge theory.

2. Similarly we consider the RS dual or mirror configuration. The world-volume theory for a threebrane stretched between two D fivebranes is that of a massless hypermultiplet. Given $n_{h}$ parallel threebranes suspended between the same two D fivebranes, we get $n_{h}$ massless hypermultiplets as long as their positions are far enough apart; what actually happens when they are close must be elucidated. By an RS duality transformation turning the D fivebranes into NS fivebranes, the hypermultiplets are converted into vectors and the $n_{h}$ massless hypermultiplets parametrize the Coulomb branch of a $U\left(n_{h}\right)$ gauge theory.

3. The last case is that of a threebrane which terminates on a D fivebrane at one end and an NS fivebrane at the other end. Such a threebrane has no moduli. $\vec{x}$ is fixed at one end to equal the $\vec{m}$ value of the D fivebrane (and its supersymmetric partner $a_{\mu}$ 
is projected out by Dirichlet boundary conditions at that end) and $\vec{y}$ is fixed (along with $b$ ) at the other end. For a single such threebrane, there are no massless modes on the worldvolume at all: the low energy theory on a single infinite threebrane is an infrared-free $U(1)$ gauge theory, and all massless modes are projected out at one end or the other. The infrared theory has a unique vacuum with a mass gap.

The gauge group arising on a threebrane stretched between two NS fivebranes will be called an electric gauge group in what follows. The gauge coupling of the electric gauge theory is easy to identify. If the $x^{6}$ values of the two NS fivebranes are $t_{1}$ and $t_{2}$ then up to a universal multiplicative constant that depends only on the coupling constant $\tau_{I I B}$ of the underlying Type IIB superstring theory, the coupling of the effective 2+1-dimensional field theory is

$$
\frac{1}{g^{2}}=\left|t_{1}-t_{2}\right| .
$$

In fact, the four-dimensional effective coupling $g_{4}$ on a threebrane is determined universally in terms of $\tau_{I I B}$. The three-dimensional effective coupling, obtained by integrating over $x^{6}$ to reduce to a three-dimensional effective Lagrangian, is

$$
\frac{1}{g^{2}}=\frac{\left|t_{1}-t_{2}\right|}{g_{4}^{2}},
$$

and this is the basis for (3.1).

Likewise, if we perform an RS duality transformation to interpret the fields on a threebrane that ends on two D fivebranes as vector multiplets, we get a gauge theory with a gauge group that we will call a magnetic gauge group. If the $x^{6}$ values of the $\mathrm{D}$ fivebranes are $z_{1}$ and $z_{2}$, then by reasoning just as above, the magnetic gauge coupling is

$$
\frac{1}{g^{2}}=\left|z_{1}-z_{2}\right| .
$$

In both (3.1) and (3.3) we omitted an overall constant; those constants are in fact equal if the Type IIB coupling is equal to the duality-invariant value $\tau_{I I B}=i$.

We now want to discuss certain singularities that can result in the appearance of additional massless hypermultiplets in the effective $2+1$-dimensional theory. First consider a solitonic or NS fivebrane such that $k_{1}$ threebranes end on it from the left and $k_{2}$ threebranes end on it from the right, as in figure 1.

We want to consider what happens when one of the "left" threebranes meets one of the "right" threebranes, that is, when their $\vec{x}$ values coincide and they actually meet in space-time. This is a sort of singularity at which extra massless states might appear. In 


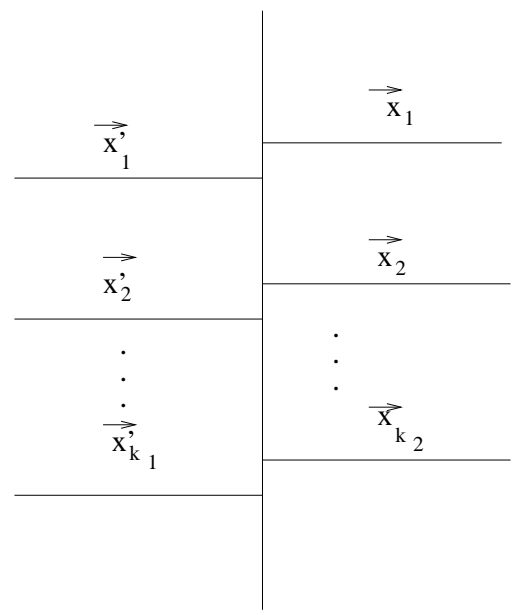

Figure 1: Here and in subsequent figures, vertical solid lines represent NS fivebranes in the 012345 directions, and horizontal lines represent 0126 threebranes. In the example depicted here, the threebranes come from left or right, and a massless hypermultiplet appears whenever a "left" and "right" threebrane meet.

this case it is easy to guess heuristically what might happen. A string stretched between the "left" threebrane and the "right" threebrane could give a hypermultiplet that becomes massless in the limit that the two threebranes meet. To be somewhat more precise, if the $\vec{x}$ 's of the two threebranes differ by a vector of length $\alpha$ that points in the $x^{3}$ direction then an elementary string stretched between the two threebranes would appear to give a BPS hypermultiplet of mass $\alpha$, invariant under supersymmetries generated by parameters that obey

$$
\epsilon_{L}=\Gamma_{0} \Gamma_{3} \epsilon_{L}, \quad \epsilon_{R}=\Gamma_{0} \Gamma_{3} \epsilon_{R},
$$

as well as equations (2.1) - (2.4). This hypermultiplet will become massless when the two threebranes meet. The existence of this state is not really a sound deduction from perturbative string theory - as the configuration of figure 1 is beyond the reach of perturbative string theory - and we will eventually give additional arguments that this state must be present.

The hypermultiplet obtained in this way contains four real scalars which transform in the $(1,2)$ representation of the R-symmetry group $S U(2)_{V} \times S U(2)_{H}$ (the representation $(1,2)$ is four-dimensional if viewed as a real representation) plus fermions. The coupling of this hypermultiplet to the "electric" vector multiplets on the threebranes gives it a mass proportional to $\left|\vec{x}_{L}-\vec{x}_{R}\right|$ where $\vec{x}_{L}$ and $\vec{x}_{R}$ are the $\vec{x}$ values of the "left" and "right" threebrane.

Now consider the full configuration of figure 1 with many $k_{1}$ "left" threebranes and 
$k_{2}$ "right" ones. As there are $k_{1} k_{2}$ possible coincidences, involving the meeting of a "left" threebrane with a "right" one, there are a total of $k_{1} k_{2}$ hypermultiplets associated with these singularities. In view of their couplings to the $U(1)$ gauge fields on the various threebranes, they clearly transform as $\left(k_{1}, \bar{k}_{2}\right)$ under $U\left(k_{1}\right) \times U\left(k_{2}\right)$.

Next let us consider the mirror configuration, in which we will get hypermultiplets that are charged with respect to the magnetic gauge group. Performing an RS duality transformation which converts NS fivebranes to D fivebranes while leaving the threebranes invariant, the picture considered above is converted to that of figure 2. In this case,

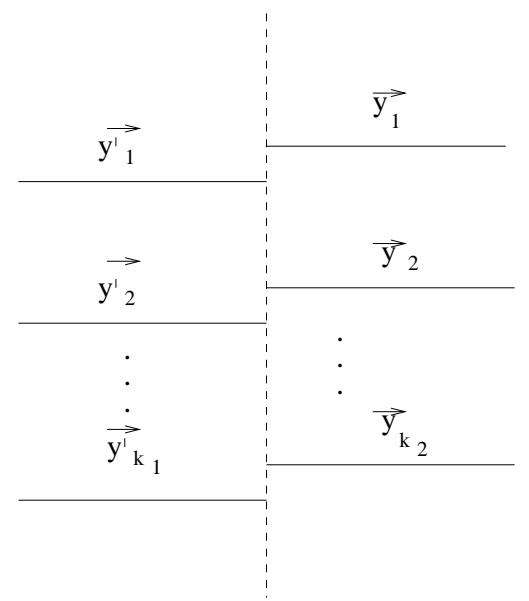

Figure 2: Here and in subsequent figures, vertical dashed lines represent D fivebranes in the 012789 directions (which one might think of as coming "out of the paper"), while horizontal lines represent D3 branes which in this example come from the left or right.

coincidences of "left" and "right" threebranes give massless hypermultiplets transforming as $\left(k_{1}, \bar{k}_{2}\right)$ of the magnetic gauge group. Under the $S U(2)_{V} \times S U(2)_{H}$ symmetry, the scalars in these hypermultiplets transform as $(2,1)$.

Notice that these two mirror-symmetric constructions gives hypermultiplets that transform differently under $S U(2)_{V} \times S U(2)_{H}$, in fact as $(1,2)$ and $(2,1)$ respectively. When it is necessary to draw a distinction, we will call the fields obtained in this way (and charged with respect to electric or magnetic gauge groups) electric hypermultiplets and magnetic hypermultiplets, respectively.

There is another situation in which hypermultiplets appear. Consider as in figure 3 a threebrane perpendicular to a D fivebrane or an NS fivebrane. A massless hypermultiplet appears when the threebrane and fivebrane actually meet in spacetime, which occurs when the threebrane transverse position is suitably adjusted. In figure 3(b), where the fivebrane is Dirichlet, this can be demonstrated explicitly in weakly coupled string theory 


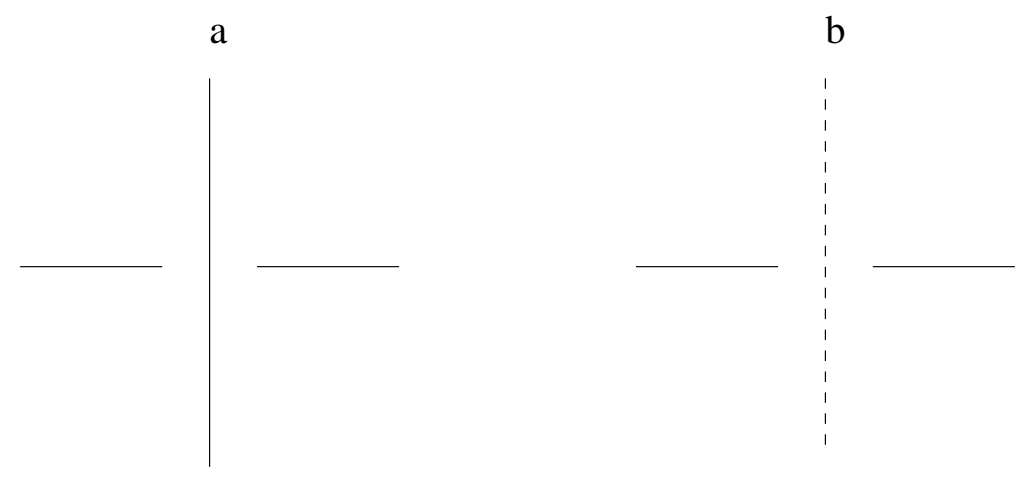

Figure 3: Electric and magnetic hypermultiplets. Sketched in figure (a) is a threebrane (horizontal line) perpendicular to an NS fivebrane (vertical line). When they actually meet in space (that is, when the $\vec{m}$ values are equal), a massless hypermultiplet appears. Sketched in figure (b) are a D fivebrane (vertical dotted line) and perpendicular threebrane (horizontal line); a massless magnetic hypermultiplet appears when they actually meet in space. In later figures, we will not be so careful in indicating whether branes represented by crossing perpendicular lines actually meet in space.

by considering an elementary Type IIB superstring suspended between the threebrane and the D fivebrane. This gives a hypermultiplet with scalar fields transforming as $(1,2)$ under $S U(2)_{V} \times S U(2)_{H}$ and mass parameters equal to $\vec{x}-\vec{m}$. By mirror symmetry, the configuration of figure 3(a), with a solitonic or NS fivebrane, therefore gives a hypermultiplet with scalars transforming as $(2,1)$ under $S U(2)_{V} \times S U(2)_{H}$ and mass parameters $\vec{y}-\vec{w}$.

Notice that we have now given two different ways of obtaining such light "electric" hypermultiplets, involving the configurations of figures 2 and 3(b). In the second, the appearance of the light hypermultiplet is a precise deduction of perturbative string theory, and in the first it is not. Later, we will understand some "phase transitions" in which the second mechanism of generating a light electric hypermultiplet is converted into the first, giving a powerful check that the situation of figure 1 behaves as claimed. One of these phase transitions is based on an esoteric process involving motion of fivebranes, but one can be described very simply. Consider as in figure 3 a threebrane perpendicular to a fivebrane, and adjust the threebrane position so that it meets the fivebrane in space; it is certainly plausible intuitively that the threebrane can then "break" into two pieces, one meeting the fivebrane from the left and one from the right, as in figure 1. If such a transition between the configurations of figures 1 and 3 is possible - and in section five we will see that such a transition is needed to reproduce certain standard field theory phenomena - then, since figure 3 generates a massless hypermultiplet at the point at 
which the different branes meet in spacetime, figure 1 must do the same.
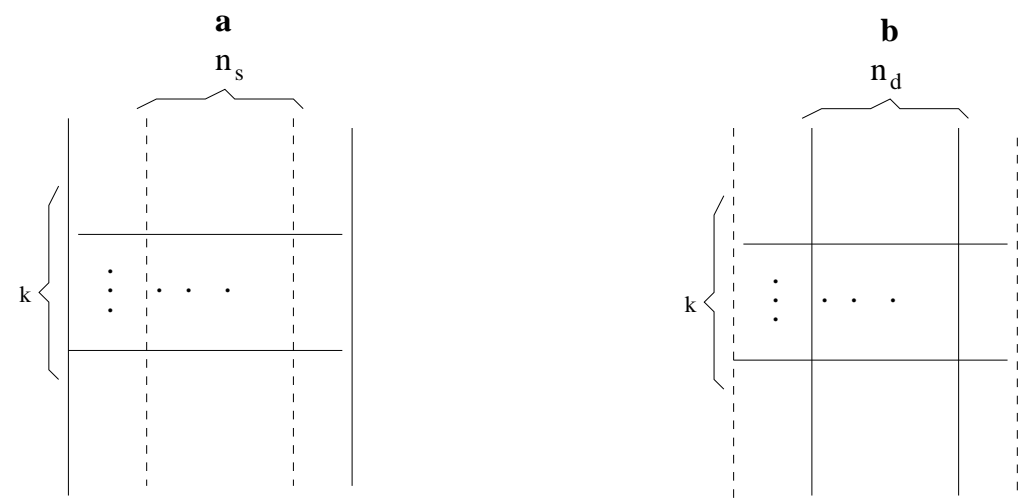

Figure 4: Hypermultiplets in electric and magnetic gauge theories. In part (a), electric hypers are given by threebrane intersections with $\mathrm{D}$ fivebranes. In part (b), magnetic hypers come from intersections NS fivebranes.

A particularly important application of the construction described in the last two paragraphs is as follows. Consider as in figure 4(a) two solitonic five branes with $x^{6}$ positions $t_{1}$ and $t_{2}$, with $t_{1}<t_{2}$, and with $k$ threebranes stretching in between them. Such a supersymmetric configuration exists if and only if the two solitonic fivebranes have $\vec{w}_{1}=\vec{w}_{2}$. Suppose that in addition there is present as well a $\mathrm{D}$ fivebrane with $x^{6}$ position $t_{1}<z<t_{2}$. Then by what we have just said, a massless hypermultiplet appears whenever a threebrane meets a $\mathrm{D}$ fivebrane, that is whenever the $\vec{x}$ value of the threebrane equals the $\vec{m}$ transverse position of the D fivebrane.

If there are $k$ parallel threebranes in figure 4(a), then the effective world-volume theory is a $U(k)$ gauge theory, and the intersections with the $\mathrm{D}$ fivebrane will give $k$ potentially massless hypermultiplets transforming in the fundamental representation of $U(k)$. Such a multiplet can have a supersymmetric bare mass, transforming as $(3,1)$ of $S U(2)_{V} \times$ $S U(2)_{H}$. In this case, the bare mass is equal to the transverse location $\vec{m}$ of the Dirichlet fivebrane. For a single hypermultiplet in the fundamental representation of $U(k)$, this bare mass is not very exciting, as it can be canceled by moving the $\vec{x}$ values of the threebranes, that is, by shifting the scalars in the $U(k)$ vector multiplet.

Suppose more generally that as in the figure there are $n_{d} \mathrm{D}$ fivebranes whose positions are $z_{i}, \vec{m}_{i}, 1 \leq i \leq n_{d}$, with $t_{1}<z_{i}<t_{2}$. Intersections with the threebranes now give hypermultiplets transforming as $n_{d}$ copies of the fundamental representation of $U(k)$. The bare mass of the $j^{\text {th }}$ such multiplet is $\vec{m}_{j}$, and the differences $\vec{m}_{i}-\vec{m}_{j}$ are observable parameters of the low energy effective theory in $2+1$ dimensions; they cannot be eliminated by shifting any of the dynamical fields. 
Since the picture of figure 4(a) corresponds to a $U(k)$ gauge theory, and $U(k)$ has a non-trivial center $U(1)$, a Fayet-Iliopoulos $D$-term for the $U(1)$ should be possible. This is simply the difference $\vec{D}=\vec{w}_{1}-\vec{w}_{2}$ between the transverse positions of the two NS fivebranes at the ends of the figure. We already noted that a supersymmetric configuration of the type sketched in figure 4 (a) only exists if $\vec{w}_{1}-\vec{w}_{2}=0$. For $\vec{D} \neq 0$, a supersymmetric vacuum may still exist, but only after making a transition to a Higgs branch such as we discuss later.

Finally, we consider the mirror of this. This is obtained, of course, by converting all NS fivebranes into D fivebranes, and vice-versa, to arrive at figure 4(b). Now we get massless hypermultiplets transforming in the fundamental representation of a magnetic $U(k)$ gauge group; the massless scalars transform as $(2,1)$ of $S U(2)_{V} \times S U(2)_{H}$, and the bare mass parameters are the $\vec{w}$ parameters of the NS fivebranes. The difference $\vec{m}_{1}-\vec{m}_{2}$ for the D fivebranes at the ends of the figure is the Fayet-Iliopoulos $D$-term for the magnetic $U(k)$ gauge theory.

\section{The Coulomb Branch And The Moduli Space Of Monopoles}

We finally move on to applications. In this section, we consider the relatively tame situation in which all gauge groups are electric. As we will see, the phenomena are still quite rich and interesting.

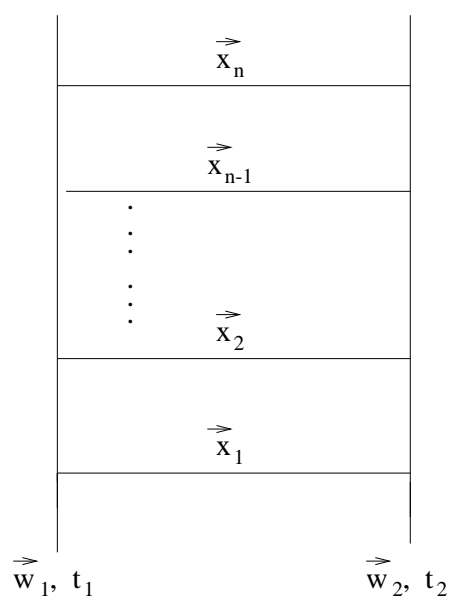

Figure 5: $S U(2)$ monopoles versus pure $N=4 U(k)$ three dimensional gauge theories. The vertical lines represent solitonic five branes at the positions $\vec{w}_{1}, t_{1}$ and $\vec{w}_{2}, t_{2}$. The horizontal lines represent D3 branes with positions $\vec{x}_{i}, i=1, \ldots, k$. 
First we consider, as in figure 5, a system of $k$ parallel threebranes suspended between a pair of NS fivebranes. To an observer on the threebranes, the low energy theory is, as we have seen in the last section, a $U(k)$ electric gauge theory with no hypermultiplets. The transverse positions of the threebranes (together with their superpartners, the scalars dual to the vector fields on the threebranes) parametrize the Coulomb branch of this theory.

On the other hand, the same configuration can be viewed in another way. A $5+1$ dimensional observer sees a $U(2)$ gauge theory of two parallel fivebranes, with $U(2)$ broken to $U(1) \times U(1)$ by the separation between these branes. Actually the center $U(1) \subset U(2)$ will play no role in this discussion - as the light fields are all neutral under this $U(1)-$ so we can work modulo the center and think of the fivebranes as carrying a $S U(2)$ gauge symmetry that is broken to $U(1)$. The Higgs field that controls this symmetry breaking is simply $t_{1}-t_{2}$, the separation between the two fivebranes in the $x^{6}$ coordinate. The fivebrane theory of $S U(2)$ broken to $U(1)$ is to be treated classically as the fivebranes are so heavy.

Now from the point of view of the fivebrane theory, the end of a threebrane looks like a magnetic monopole. (To be more precise, this might be called a magnetically charged twobrane; a magnetic monopole is a particle in $3+1$ dimensions or a twobrane in $5+1$ dimensions. However, we will be treating the fivebrane theory classically and our configurations will all be invariant under translations of $x^{1}$, and $x^{2}$, so it is reasonable to call them magnetic monopoles in a $3+1$-dimensional reduction of the fivebrane worldvolume in which $x^{1}$ and $x^{2}$ are suppressed.) Since in figure 5 we have $k$ threebranes ending on the fivebranes, the configuration looks like one of magnetic charge $k$ from the point of view of the fivebrane observer.

Because of the unbroken supersymmetry of the brane configuration in figure 5, the fivebrane observer sees, to be more precise, a BPS-saturated configuration with magnetic charge $k$ which depends on a total of $4 k$ real moduli - the same parameters (threebrane transverse positions and scalars dual to threebrane world-volume vectors) which we earlier interpreted as parametrizing the Coulomb branch of the $U(k)$ gauge theory. These $4 k$ variables parametrize a hyper-Kahler manifold $\mathcal{M}$.

Thus, we have something that may sound familiar: a gauge theory with $S U(2)$ broken to $U(1)$, and a $4 k$-dimensional hyper-Kahler manifold parametrizing BPS-saturated configurations of magnetic charge $k$. The classical moduli space $\mathcal{M}_{c l}$ of BPS monopoles of magnetic charge $k$ has exactly these properties [13], and it is natural to suspect that $\mathcal{M}=\mathcal{M}_{c l}$. In fact, essentially this question has been analyzed by Diaconescu [14]. After making a mirror transformation to convert the NS fivebranes of figure 5 to D fivebranes, 
followed by a $T$-duality transformations to reduce to onebranes ending on threebranes, the configuration of figure 5 turns into the system of strings ending on threebranes considered by Diaconescu, who analyzed the string dynamics and obtained the Nahm equations for monopoles, thus showing that $\mathcal{M}=\mathcal{M}_{c l}$.

So at this point, we have learned that one moduli space - that of figure 5 - can be looked at in two ways. It is the Coulomb branch of the pure $U(k)$ quantum gauge theory in three dimensions, without hypermultiplets, or it is the moduli space of $k$ monopoles in a classical gauge theory in three dimensions with $S U(2)$ broken down to $U(1)$. This correspondence has been previously noted in [1] for $k=2$ and in [2] for general $k$.

Notice that, as the center $U(1) \subset U(k)$ decouples from the $U(k)$ gauge theory, the moduli space considered here is a product of the Coulomb branch of an $S U(k)$ gauge theory with the moduli space of a free vector multiplet. From the other point of view, the free vector multiplet parametrizes the center of mass of the $k$-monopole configuration. An alternative statement is thus that the reduced moduli space of the $k$-monopole system, with the center of mass position factored out, is the Coulomb branch of the supersymmetric $S U(k)$ gauge theory with no hypermultiplets.

\subsection{The General Case}

Now we will consider a much more general situation of the same kind.

We consider a $U(n)$ gauge theory with $U(n)$ broken to $U(1)^{n}$ by the expectation value of a Higgs field $\phi$ which takes values in the adjoint representation; that is, $\phi$ is an $n \times n$ Hermitian matrix. The symmetry breaking is parametrized by the eigenvalues of $\phi$ which we call $\phi_{i}$, with $\phi_{1}<\phi_{2}<\cdots \phi_{n}$. (We could replace $U(n)$ by $S U(n)$ and add a constant to $\phi$ so that $\sum_{i} \phi_{i}=0$; this would bring no essential change in what follows.)

A basic $S U(2)$ BPS monopole can be embedded in $U(n)$ in $n-1$ different ways, to give monopoles of magnetic charge $(1,-1,0, \ldots, 0),(0,1,-1, \ldots, 0), \ldots$, or $(0,0, \ldots, 1,-1)$. By combining $k_{i}$ monopoles of the $i^{\text {th }}$ type for $1 \leq i \leq n-1$ we build monopoles of charges $\left(k_{1}, k_{2}-k_{1}, \ldots,-k_{n}\right)$ and the general BPS configuration is parametrized by the choice of the non-negative integers $k_{i}$ as well as of the Higgs eigenvalues $\phi_{i}[15,16]$.

Now we can realize this general component of $U(n)$ monopole moduli space via a brane configuration, as follows. We consider, as in figure $6, n$ parallel NS fivebranes, with $x^{6}$ values $t_{1}, \ldots, t_{n}$, and with the $i^{\text {th }}$ fivebrane connected to the $i+1^{\text {th }}$ by $k_{i}$ threebranes. Such a configuration is possible precisely if the fivebranes have a common value of $\vec{w}$, so the parameters that enter in defining it are the continuous variables $t_{j}$ and the integers 


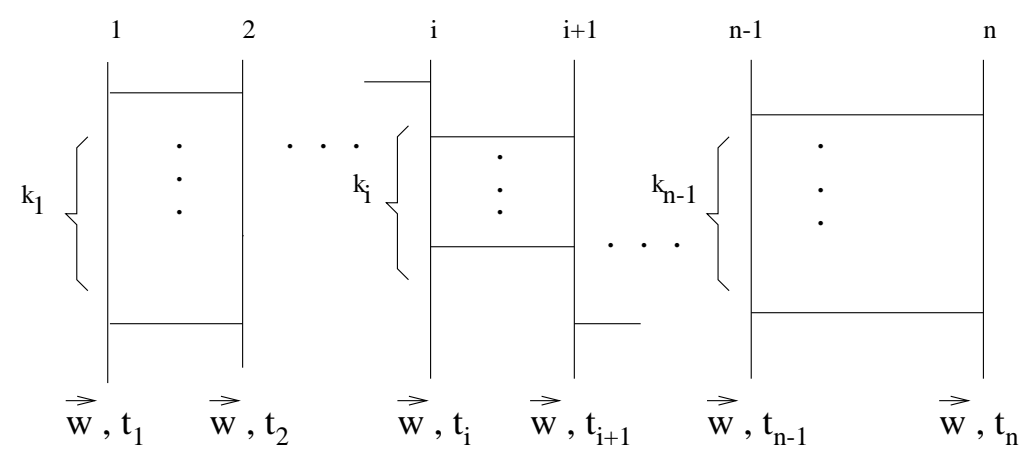

Figure 6: $S U(n)$ monopoles versus three dimensional gauge theories.

$k_{j}$

Looked at from the fivebrane point of view, the configuration of figure 6 is a $U(n)$ monopole, with Higgs eigenvalues $\phi_{j}=t_{j}$, and magnetic charges given by the $k_{j}$. The moduli space is thus the BPS moduli space of such objects, which we will call $\mathcal{M}\left(k_{j} ; \phi_{j}\right)$. On the other hand, from the threebrane point of view, we have macroscopically a $2+1$ dimensional supersymmetric gauge theory with gauge group $U\left(k_{1}\right) \times U\left(k_{2}\right) \times \cdots \times U\left(k_{n-1}\right)$ and with, in an obvious notation, hypermultiplets of zero bare mass transforming in the representation $\left(\mathbf{k}_{\mathbf{1}}, \overline{\mathbf{k}}_{\mathbf{2}}\right) \oplus\left(\mathbf{k}_{\mathbf{2}}, \overline{\mathbf{k}}_{\mathbf{3}}\right) \oplus \cdots \oplus\left(\mathbf{k}_{\mathbf{n}-\mathbf{2}}, \overline{\mathbf{k}}_{\mathbf{n}-\mathbf{1}}\right)$. In this gauge theory, the gauge coupling of the $j^{\text {th }}$ factor $U\left(k_{j}\right)$ of the gauge group is given by

$$
\frac{1}{g_{j}^{2}}=\left|\phi_{j}-\phi_{j+1}\right| \text {. }
$$

The Coulomb branch of this gauge theory must thus coincide, as a hyper-Kahler manifold, with the BPS moduli space $\mathcal{M}\left(k_{j} ; \phi_{j}\right)$. Thus, we have identified all BPS moduli spaces for $U(n)$ gauge group with the Coulomb branches of suitable gauge theories.

As a special case of this, suppose that there are precisely three fivebranes, so that the gauge group is $U\left(k_{1}\right) \times U\left(k_{2}\right)$ and the hypermultiplets transform as $\left(\mathbf{k}_{\mathbf{1}}, \overline{\mathbf{k}}_{\mathbf{2}}\right)$. The gauge couplings of $U\left(k_{1}\right)$ and $U\left(k_{2}\right)$ are respectively

$$
\frac{1}{g_{1}^{2}}=t_{2}-t_{1}=\phi_{2}-\phi_{1}
$$

and

$$
\frac{1}{g_{2}^{2}}=t_{3}-t_{2}=\phi_{3}-\phi_{2}
$$

The two gauge couplings are completely independent, and if we wish we can take $g_{2} \rightarrow 0$, keeping $g_{1}$ fixed, by taking $\phi_{3} \rightarrow \infty$, with $\phi_{2}-\phi_{1}$ fixed. In the limit that $g_{2}$ is turned off, the theory, from the threebrane point of view, turns into a $U\left(k_{1}\right)$ gauge theory with 
hypermultiplets transforming as $k_{2}$ copies of the fundamental representation of $U\left(k_{1}\right)$; the $U\left(k_{2}\right)$ gauge symmetry reduces to a global $U\left(k_{2}\right)$ symmetry acting on $k_{2}$ multiplets. The hypermultiplets have arbitrary bare masses determined by the expectation values of the scalars in the $U\left(k_{2}\right)$ vector multiplet.

Now, let us look at it from the fivebrane point of view. Here we are dealing with $S U(3)$ monopoles. There are two basic $S U(3)$ monopoles, of magnetic charge $(1,-1,0)$ and $(0,1,-1)$. The sizes of the two monopoles are proportional to $1 /\left|\phi_{2}-\phi_{1}\right|$ and $1 /\left|\phi_{3}-\phi_{2}\right|$, respectively. Thus when $\phi_{3}$ becomes very large with fixed $\phi_{2}-\phi_{1}$, the $(0,1,-1)$ shrinks while the $(1,-1,0)$ monopole retains a fixed size. In our problem, we have $k_{1}$ of the $(1,-1,0)$ monopoles combined with $k_{2}$ of the $(0,1,-1)$ monopoles. In the limit that $\phi_{3} \rightarrow \infty$ with $\phi_{2}-\phi_{1}$ fixed, one would expect the $(0,1,-1)$ monopoles to shrink to point singularities, so we should be left with an $S U(2)$ monopole solution of magnetic charge $k_{1}$ with $k_{2}$ point singularities. The moduli space of such singular $S U(2)$ monopoles should coincide with the Coulomb branch of the $U\left(k_{1}\right)$ gauge theory with hypermultiplets transforming as $k_{2}$ copies of the fundamental representation. The positions of the singularities correspond to the bare masses of the hypermultiplets in the field theory.

Kronheimer [17] constructed a theory of $S U(2)$ monopoles with point singularities and showed a close relation of the data involved to the data parametrizing $S U(3)$ monopoles. Very plausibly, the singular monopoles considered by Kronheimer are the ones relevant here.

\section{Explicit Comparison To Field Theory}

It should be possible to test the above claims explicitly by comparing computations of the metric on monopole moduli space in the region in which the monopoles are widely separated to computations of instanton corrections to the Coulomb branch of the field theory. The instantons in question are actually monopoles (of the threebrane quantum gauge theory!), and can be reinterpreted in string theory in the following somewhat subtle way.

As a simple relevant example, consider the case that the fivebrane description involve $S U(2)$ monopoles of magnetic charge two, and the three-dimensional quantum gauge theory is an $S U(2)$ gauge theory without hypermultiplets. The corresponding string theory configuration consists of two parallel NS fivebranes and two threebranes stretching between them. The two-monopole moduli space of $S U(2)$ is the Atiyah-Hitchin manifold, studied in detail in [13]; its metric differs from a Taub-NUT metric by terms that vanish exponentially at infinity. We wish to interpret the exponentially small corrections. 
According to [1], these corrections are due to monopoles, regarded as instantons of the $2+1$-dimensional theory, and make contributions proportional to $e^{-(I+i \sigma)}$, where $I$ is the action of the instanton and $\sigma$ is the scalar dual to the $U(1)$ photon. The action $I$ is proportional to $\frac{|\vec{x}|}{g^{2}}$ where $\vec{x}$ are the scalars in the vector multiplet while $g$ is the three dimensional coupling. We need to look for a string theory instanton which give this action. This action is nothing but the area spanned between the two D3 branes as in figure 7 .

We thus claim a slight enrichment of the general picture of branes ending on branes. Not only can a D onebrane worldsheet end on a threebrane or a fivebrane; it can also have a "corner" where the threebrane ends on a fivebrane. Such onebrane worldvolumes with boundaries and corners are needed to reproduce in string theory the field theory instanton corrections to the metric on the two-monopole moduli space.

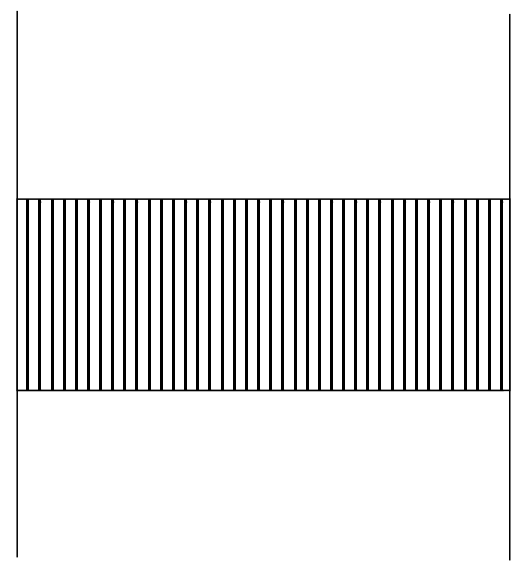

Figure 7: Instanton corrections to the metric on the Coulmb branch in $S U(2)$ gauge theory. These corrections come from strings stretched between the two D3 branes. The shaded region is two-dimensional and is localized at specific values of $x^{0}, x^{1}$, and $x^{2}$ (the instanton position).

\subsection{A Special Example}

Finally, we consider in more detail another special example.

If all $k_{i}$ equal one, then the threebrane gauge theory is abelian, with gauge group $U(1)^{n-1}$. The theory contains $n-2$ charged hypermultiplets, of charges $(1,-1,0, \ldots, 0)$, $(0,1,-1,0, \ldots, 0), \cdots,(0,0, \ldots, 0,1,-1)$. Notice that the sum of the charges is zero for each hypermultiplet, so after factoring out from the gauge group a decoupled $U(1)$, this is a $U(1)^{n-2}$ gauge theory with $n-2$ charged hypermultiplets.

For instance, the first case $n=3$ is the $U(1)$ theory with one charged hypermultiplet, 
which was shown in [1] to have for its Coulomb branch a smooth Taub-NUT manifold. This indeed agrees, as it should, with the $S U(3)$ monopole space for monopoles of charge $(1,0,-1)$. This manifold was first studied in [18] and later discussed in $[19,20]$.

The fact that the gauge group is abelian means that there are no instantons in the $2+1$-dimensional field theory. Consequently, the metric of the models with all $k_{i}=1$ should be given exactly by a one-loop formula, with no exponentially small corrections, and should be invariant under shifts of the scalars dual to the photons.*

The Taub-NUT metric has these properties.

More generally, for arbitrary $n$, the Coulomb branch of this theory should coincide with the moduli space of $S U(n)$ monopoles of magnetic charge $(1,0, \ldots, 0,-1)$. The features reflecting absence of instanton corrections have indeed been found in recent studies. The metric on the moduli space of $(1,0,0, \ldots,-1)$ monopoles was conjectured in [23] and later proved by $[16,24]$ to be given by the formulas

$$
\begin{gathered}
d s^{2}=g_{i j} d \vec{x}_{i} \cdot d \vec{x}_{j}+\left(g^{-1}\right)_{i j} d \tilde{\theta}_{i} d \tilde{\theta}_{j}, \\
d \tilde{\theta}_{i}=d \theta_{i}+\sum_{j=1}^{n} \vec{W}_{i j} \cdot d \vec{x}_{j} .
\end{gathered}
$$

Here the function $\vec{W}_{i j}$ is the potential arising from a Dirac point monopole at the point $i$ acting at a monopole at the point $j$.

$$
\begin{gathered}
W_{i i}=\sum_{i \neq j} w_{i j}, \quad W_{i j}=-w_{i j}, \quad i \neq j . \\
g_{i i}=\left|t_{i}-t_{i+1}\right|+\frac{g^{2}}{4 \pi} \sum_{i \neq j} \frac{1}{\left|\vec{x}_{i}-\vec{x}_{j}\right|} \quad g_{i j}=-\frac{1}{\left|\vec{x}_{i}-\vec{x}_{j}\right|}, \quad i \neq j .
\end{gathered}
$$

This formula can be seen by symmetry arguments to agree with the Coulomb branch of the $U(1)^{n-1}$ gauge theory with the one loop correction. The decoupled $U(1)$ factor in $U(1)^{n-1}$ corresponds as usual to the overall translational degree of freedom of the monopole system. The metric just given can be interpreted as obtained from a flat metric by a one-loop correction; the extra symmetries that arise because the threebrane theory has no instantons are simply the constant shifts in the $\theta$ 's.

Incidentally, the bound state (or $\mathbf{L}^{2}$ harmonic form) on this particular $S U(n)$ monopole moduli space that is predicted by duality and whose existence has recently been confirmed,

${ }^{*}$ These facts are actually related in the following way. The invariance of the metric under shifts in the scalars actually means that the Coulomb branch admits what is called a tri-holomorphic torus action of dimension equal to the number of vector multiplets. This then implies [21,22] that the metric can be written in a relatively elementary form using solutions of linear equations. 
for the case $n=3$ by $[19,20]$ and for general $n$ by [25], plausibly corresponds in the threebrane language to a situation in which a single threebrane stretches all the way between the leftmost and rightmost fivebranes, without being split up into pieces.

\section{A First Look At Nontrivial Phase Trasitions}

Our aim is to incorporate both NS and D fivebranes and study the mirror symmetry that exchanges them. First, though, we must explain a new aspect of brane interactions that is needed in order to study processes in which both kinds of fivebrane are present.

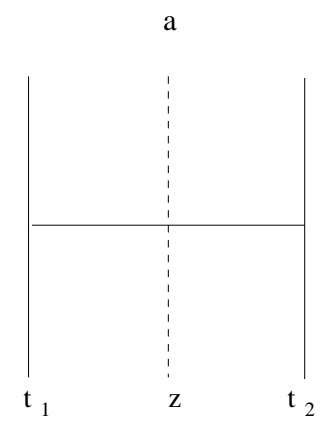

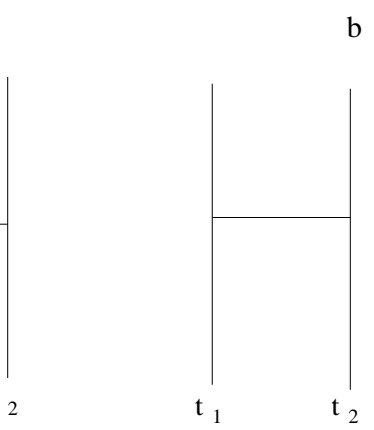

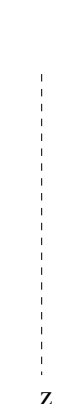

c

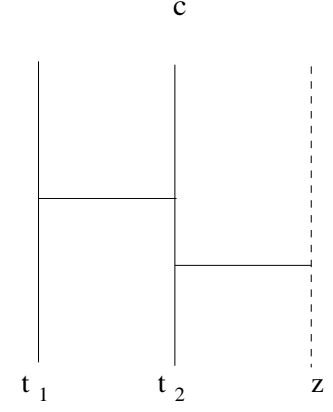

Figure 8: Two fivebranes cross each other. In figure (a) the starting configuration. In figure (b) the naive configuration obtained by moving the D fivebrane of (a) to the right; consideration of this transition leads to a paradox. In figure (c) the correct configuration.

To see the need for a new phenomenon, we first describe an apparent contradiction. In figure 8 we sketch two NS fivebranes at $t_{1}, \vec{w}_{1}$ and $t_{2}, \vec{w}_{2}$, and a D fivebrane at $z, \vec{m}$. There also is a threebrane connecting the two NS fivebranes, whose transverse position in the $3-4-5$ directions is $\vec{x}$.

First we consider the case that $t_{1}<z<t_{2}$, as in figure 8(a). In this figure there is a BPS hypermultiplet - from strings connecting the threebrane to the D fivebrane - with a mass proportional to $|\vec{m}-\vec{x}|$.

Now gradually increase $z$ to get to the situation $t_{1}<t_{2}<z$. Superficially, the system should then be as depicted in figure 8(b). Here, though, there is a paradox: in figure 8(b) there is no reason to get any massless hypermultiplet (or any other singularity) at $\vec{m}-\vec{x}=0$. In general, in models with three-dimensional $N=4$ (or four-diemnsional $\mathcal{N}=2$ ) supersymmetry, the BPS spectrum can jump, but in the specific case at hand, there is nothing for the hypermultiplet in question to decay to. 
The resolution that we propose for this seeming paradox is as follows. Note that at $z=t_{2}$, the $\mathrm{D}$ and NS fivebranes actually meet in spacetime. In fact, the D fivebrane is parametrized by the values of $x^{0}, x^{1}, x^{2}, x^{7}, x^{8}, x^{9}$, with constant values of the other coordinates. The NS fivebrane is parametrized by the values of $x^{0}, x^{1}, x^{2}, x^{3}, x^{4}, x^{5}$ with constant values of the others. For them to meet in spacetime it is therefore necessary and sufficient that the $x^{6}$ values coincide, and this in particular occurs at $z=t_{2}$.

Thus, in trying to deform figure 8(a) to figure 8(b), the two fivebranes have "passed through" each other. Our proposal is that when the two branes pass through each other, a third brane is created. In fact, we claim that figure 8(a) deforms not to figure 8(b) but to figure 8(c). In figure 8(c), in addition to the threebrane that was present in $8(\mathrm{a})$, there is a new threebrane, running from the NS fivebrane at $x^{6}=t_{2}$ to the D fivebrane at $x^{6}=z$. This new threebrane, since it connects an NS fivebrane to a D fivebrane, has no moduli; its $\vec{x}$ is the $\vec{m}$ of the D fivebrane, and its $\vec{y}$ is the $\vec{w}$ of the NS fivebrane. Thus, the moduli of figure $8(\mathrm{c})$ are the same as those of figure $8(\mathrm{a})$ or figure $8(\mathrm{~b})$.

The virtue of this proposal is that in figure $8(\mathrm{c})$, unlike figure $8(\mathrm{~b})$, there is a mechanism to get a massless hypermultiplet whenever $\vec{x}=\vec{m}$. Precisely under this condition, the two threebranes in figure 8(c) meet in spacetime, and an elementary string stretched between them should plausibly give a massless hypermultiplet.

In fact, the mechanism for getting a massless hypermultiplet in figure 8(a) is a special case of the mechanism in figure 3(b), while the mechanism for getting a massless hypermultiplet in figure $8(\mathrm{c})$ is a special case of the mechanism in figure 1. So as promised in section two, we have obtained (if our resolution of the paradox can be justified) a phase transition in which one mechanism for generating a massless hypermultiplet is converted to another.

\section{A Priori Argument}

Now we will give an a priori argument that the phenomenon invoked in our resolution of the paradox must actually occur.

Let $X_{N S}$ be the world-volume of an NS fivebrane, and let $X_{D}$ be the world-volume of a D fivebrane. First of all, the NS fivebrane is the source of a three-form field strength $H_{N S}$ which has the property that if $S$ is a small three-sphere wrapping once around $X_{N S}$, then

$$
\int_{S} \frac{H_{N S}}{2 \pi}=1
$$

Likewise, the D fivebrane is the source of a three-form field strength $H_{D}$ such that if $S^{\prime}$ 
is a small three-sphere wrapping once around $X_{D}$, then

$$
\int_{S^{\prime}} \frac{H_{D}}{2 \pi}=1
$$

Let us consider Type IIB superstring theory on $\mathbf{R}^{3} \times M_{7}$, with $M_{7}$ some seven-manifold. Consider the case that $X_{N S}=\mathbf{R}^{3} \times Y_{N S}$ and $X_{D}=\mathbf{R}^{3} \times Y_{D}$, where $Y_{N S}$ and $Y_{D}$ are three-manifolds in $M_{7}$. In our actual application $M_{7}=\mathbf{R}^{7}$, and $Y_{N S}$ (parametrized by $x^{3}, x^{4}, x^{5}$ ) and $Y_{D}$ (parametrized by $x^{7}, x^{8}, x^{9}$ ) are copies of $\mathbf{R}^{3} \subset \mathbf{R}^{7}$. But temporarily we do not specify $M_{7}$, and we take $Y_{N S}$ and $Y_{D}$ to be compact and disjoint three-manifolds in $M_{7}$. In this situation, under a mild topological restriction $\left(Y_{N S}\right.$ and $Y_{D}$ should be trivial in $H_{3}\left(M_{7}, \mathbf{Z}\right)$ ) one can define the "linking number" of the three-manifolds $Y_{N S}$ and $Y_{D}$ in the seven-manifold $M_{7}$. Let $H_{N S}$ be the $H$-field created by the NS fivebrane on $\mathbf{R}^{3} \times Y_{N S}$, and let $H_{D}$ be the $H$-field created by the $\mathrm{D}$ fivebrane on $\mathbf{R}^{3} \times Y_{D}$. The linking number is defined by

$$
L\left(Y_{N S}, Y_{D}\right)=\int_{Y_{D}} \frac{H_{N S}}{2 \pi}
$$

or equivalently by

$$
L\left(Y_{N S}, Y_{D}\right)=-\int_{Y_{N S}} \frac{H_{D}}{2 \pi} .
$$

(The integrals are taken at any fixed point $p \in \mathbf{R}^{3}$.)

Now locally one can write $H_{N S}=d B_{N S}$ where $B_{N S}$ is a two-form potential. If $B_{N S}$ were gauge-invariant and hence globally defined, it would follow that the integral in (5.3) would vanish. In general, in Type IIB superstring theory, $B_{N S}$ is not gauge-invariant and is only defined locally. Something special happens, however, on the world-volume of a D fivebrane. On the fivebrane, there is a $U(1)$ gauge field $A_{D}$, of two-form field strength $F_{D}$, which "mixes" with the bulk two-form $B_{N S}$, in such a way that $\Lambda_{D}=B_{N S}-F_{D}$ is gauge invariant. Since in the absence of additional branes

$$
d F_{D}=0
$$

we have when no other branes are present a global representation of $H_{N S}$ restricted to $Y_{D}$ as the exterior derivative of a gauge-invariant field, namely

$$
\left.H_{N S}\right|_{Y_{D}}=d \Lambda_{D}
$$

When this is inserted in (5.3), we seem to learn that the linking number $L\left(Y_{N S}, Y_{D}\right)$ must vanish. To be more precise, since what appears in (5.6) is the total $H_{N S}$ due to all sources, this argument would show the total linking number of $Y_{D}$ with respect to all NS fivebranes adds up to zero. 
If we start with a situation with just one NS fivebrane and one D fivebrane of linking number zero, this would seem to imply that they cannot "pass through each other" and change the linking number. That would mean that figure 8(a) can be converted neither to figure 8(b) nor to figure 8(c). The two types of fivebrane are infinitely strong and cannot interpenetrate! Rather than this bizarre interpretation, there is a more straightforward possibility. Consider adding a threebrane whose world-volume is $\mathbf{R}^{3} \times C$, where $C$ is a curve in $M_{7}$, and suppose that the threebrane ends on $X_{D}$, that is that $C$ ends on the three-manifold $Y_{D}$. Let $p$ be the point on $Y_{D}$ at which $C$ ends. The boundary of a threebrane looks like a magnetic source on $X_{D}$, in the sense that (5.5) must be modified to read

$$
d F_{D}= \pm \delta(p)
$$

where the sign depends on the orientation, that is, on whether $C$ "begins" or "ends" on $Y_{D}$. (This basic fact, that a threebrane boundary looks like a magnetic charge on the fivebrane, was of course one of the starting points in section two.) When this effect is included, the relation (5.6) implies not that $\left.H_{N S}\right|_{Y_{D}}=d \Lambda_{D}$, but that

$$
\left.H_{N S}\right|_{Y_{D}}=d \Lambda_{D}-\sum_{\alpha} \epsilon_{\alpha} \delta\left(p_{\alpha}\right)
$$

where the $p_{\alpha}$ are points at which threebranes end, and $\epsilon_{\alpha}= \pm 1$ are the corresponding signs. When this equation is put in formula (5.3) for the linking number, we learn not that the linking number vanishes; rather, what vanishes is the sum of the linking number of $Y_{D}$ (with respect to all NS fivebranes) plus the net number of threebranes ending on $Y_{D}$.

Therefore, when an NS fivebrane passes through a D fivebrane (so that the linking number changes) a threebrane connecting them is created. This is the claim that was made above in figure $8(\mathrm{c})$.

By now we have explained the basic phenomenon, but we still need to adapt the discussion to the noncompact situation in which $M_{7}=\mathbf{R}_{7}$, and $Y_{N S}$ and $Y_{D}$ are both copies of $\mathbf{R}^{3}$. The main change is that the integral $\int_{Y_{D}} d \Lambda_{D}$ need no longer vanish; rather, it is determined by the behavior of $\Lambda_{D}$ near infinity, and is a "total magnetic charge" as seen by the fivebrane observer. This total magnetic charge need not vanish (it certainly did not vanish in the models considered in section three!) but because it can be measured at infinity it is conserved, and unchanged when we move fivebranes around.

So in our problems, to each fivebrane $X_{N S}$ or $X_{D}$ we will assign a "total magnetic charge," which will be conserved in all phase transitions. The total magnetic charge on a given fivebrane, say $X_{D}$, is the sum of the linking numbers of $X_{D}$ with respect to all NS 
fivebranes, plus the net number of threebranes ending on $X_{D}$. However, because of the noncompactness, we need some care in defining what we mean by the linking numbers. Let us go back to figure $8(\mathrm{a})$. We let $X_{N S}$ be the world-volume of the NS fivebrane on the right, and $X_{D}$ be the world-volume of the D fivebrane. We want to know their linking numbers, defined by a flux integral as in (5.3) or (5.4) above. Instead of actually evaluating integrals, we can note the following two facts.

1. By symmetry the linking integral changes sign when the relative position of the two branes in the $x^{6}$ direction is reversed.

2. It changes by +1 when they pass through each other.

These properties imply that the linking numbers, defined by the flux integrals, are $\pm 1 / 2$ depending on which brane is on the left. (The integrals (5.3) and (5.4) would always give integers when the manifolds involved are compact, but not in the noncompact situation considered here.)

The signs can be stated precisely as follows. Let $X_{N S}$ be a particular NS fivebrane. Let $r$ be the number of $\mathrm{D}$ fivebranes to the right of $X_{N S}$ and $l$ the number to the left. Let $R$ be the number of threebranes which end to the right ${ }^{*}$ of $X_{N S}$ and $L$ the number of threebranes that end to the left of $X_{N S}$. Then the total magnetic charge measured on $X_{N S}$ is

$$
L_{N S}=\frac{1}{2}(r-l)+(L-R) .
$$

The same formula holds for a $\mathrm{D}$ fivebrane $X_{D}$; if $r$ and $l$ are now the numbers of NS fivebranes on the right and left and $R$ and $L$ are the numbers of threebranes ending to the right and the left, then

$$
L_{D}=\frac{1}{2}(r-l)+(L-R) .
$$

We will also call the total magnetic charge measured on a fivebrane the total linking number of that brane (with respect to all other fivebranes and threebranes). From the above formulas, it follows that the total linking number, summed over all branes, is zero. This is the only restriction on the linking numbers for a configuration to exist, although requiring unbroken supersymmetry can impose further restrictions.

As we will see, this process of creation of a threebrane by moving fivebranes through each other has far-reaching consequences for the three-dimensional dynamics.

\footnotetext{
${ }^{*}$ To avoid any possible confusion, if $X_{N S}$ is at $x^{6}=t$, then a threebrane whose world-volume has $x^{6}>t$ ends to the right of $X_{N S}$ and one whose world-volume has $x^{6}<t$ ends to the left.
} 
There is an analogous process in $M$-theory, in which two fivebranes crossing each other become connected by a twobrane. It can be deduced in a similar fashion.

\section{$5.1 \quad U(1)$ With One Electron}

As an ilustration of this effect of threebrane creation, we consider the simplest configuration in which it happens. In this example we take the numbers of NS and D fivebranes to be $n_{s}=2, n_{d}=1$ and look at the possible theories which emerge from this configuration. The parameters in the theory are two solitonic five brane locations $\vec{w}_{1}, \vec{w}_{2}$, their singlet superpartners $t_{1}, t_{2}$ and the D5 brane locations $\vec{m}$ and $z$. These parameters are thought of as background fields (or coupling constants) for the three dimensional gauge theory. We can set the sum of the parameters for the solitonic five branes to zero $\vec{w}_{1}=-\vec{w}_{2}=\vec{w}$, $t_{1}=-t_{2}=t$ by a choice of origin. Similarly we can take the position of the D5 brane to be at the origin $\vec{m}=0$. We can not fix $z$ since the origin in the $x^{6}$ direction was already chosen.

We complete the specification of the model by saying that the total magnetic charges or linking numbers are to be as follows: $-1 / 2$ and $1 / 2$ for the two NS fivebranes, and 0 for the D fivebrane.

We have two phases to consider, depending on the arrangement of the fivebranes. First let us take the D5 brane to be between the two solitonic five branes, in the sense that $t_{1}<z<t_{2}$. The behavior further depends on whether (a) $\vec{w}=0$ or (b) $\vec{w} \neq 0$. In case (a), a configuration with the promised linking numbers can be built as in figure 9 (a), with a threebrane stretched between the two fivebranes. According to our rules in section three, the effective gauge theory in $2+1$ dimensions is then a $U(1)$ gauge theory with one charged hypermultiplet. Moreover, we learned in section three that the Fayet-Iliopoulos $D$-term coupling is $\vec{w}$. The transverse position or $\vec{x}$ value of the threebrane in figure $9(\mathrm{a})$ parametrizes the Coulomb branch (together with the dual of the photon).

Now we consider case (b), that is $\vec{w} \neq 0$. The field theory of $U(1)$ with one hypermultiplet and a $D$-term exhibits a Higgs mechanism; there is a unique supersymmetric vacuum, with a mass gap. Let us see how this occurs in the present context. For $\vec{w} \neq 0$, we cannot in a supersymmetric fashion suspend a threebrane between the two fivebranes. However, we can build the configuration of $9(\mathrm{~b})$, which has the same linking numbers. In this configuration, there are no moduli, since all threebranes connect fivebranes of opposite type. So this agrees with the field theory result that in the presence of the FI coupling the vacuum is unique. Note that to make an actual transition from 9(a) to 9(b), the threebrane of figure 9 (a) must meet the D fivebrane in spacetime, which occurs only 

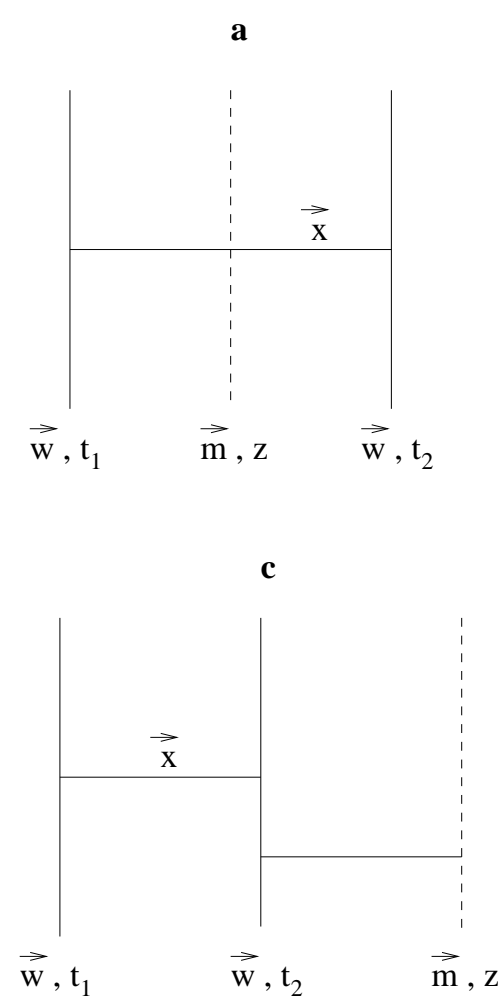

b
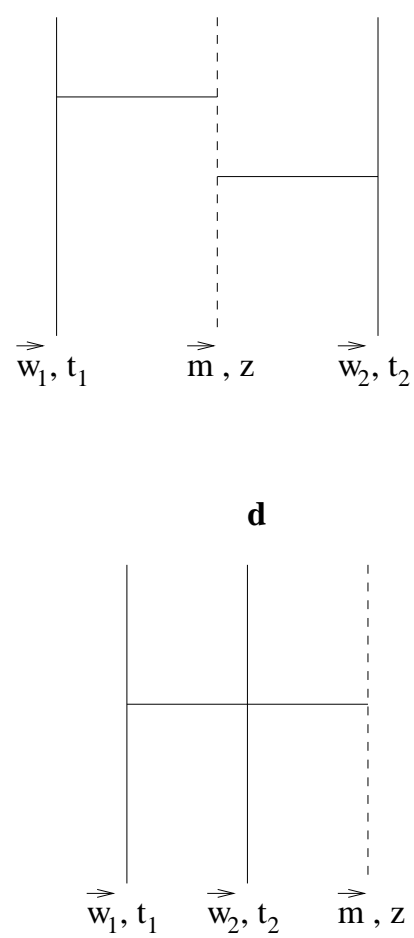

Figure 9: $U(1)$ gauge theory with one electron. In (a) and (c), $\vec{w}_{1}=\vec{w}_{2}$, but not in (b) and (d), where an FI coupling has been turned on.

at $\vec{x}=0$, that is, at the origin of the Coulomb branch. This again agrees with the field theory result.

In either case the $U(1)$ gauge coupling is $g^{2}=1 /\left|t_{2}-t_{1}\right|$. More puzzling is the interpretation of the parameter $z$ in the low energy theory. There is no obvious dependence of the low energy physics on $z$. What happens if we increase $z$ so that $z>t_{2}$ (or equivalently reduce $z$ so that $\left.z<t_{1}\right)$ ?

For $t_{1}<t_{2}<z$, the supersymmetric configurations with the given linking numbers are sketched in figure $9(\mathrm{c}, \mathrm{d})$. The case of $\vec{w}=0$ is in figure $9(\mathrm{c})$. Here we see the same spectrum as in figure $9(\mathrm{a})$ : a $U(1)$ vector multiplet with one charged hypermultiplet; its mass parameter is still $\vec{x}$ so a singularity would have to be at $\vec{x}=0$. The case of $\vec{w} \neq 0$ is in figure $9(\mathrm{~d})$. There is a single threebrane that stretches between fivebranes of opposite type, and so supports no massless modes or moduli. This again agrees with figure $9(\mathrm{~b})$.

In this model, the phase structure seems to be entirely independent of $z$ (though we cannot exclude the possibility that something exotic may happen at $z=t_{2}$ or $z=t_{1}$, perhaps if also $\vec{x}=0$ ). The parameter $z$ appears to be an irrelevant, though mysterious, 
perturbation.

It is amusing to write the metric on the Coulomb branch (which is a smooth Taub-NUT metric) in these variables. It is

$$
\begin{aligned}
d s^{2} & =G(\vec{x}) d \vec{x} \cdot d \vec{x}+G(\vec{x})^{-1} d \tilde{\theta} d \tilde{\theta}, \quad d \tilde{\theta}=d \theta-\vec{\omega} \cdot d \vec{x}, \\
G(\vec{x}) & =\left|t_{1}-t_{2}\right|+\frac{1}{|\vec{x}|}, \quad \nabla_{a} \times \vec{\omega}=\nabla_{a}\left(\frac{1}{|\vec{x}|}\right) .
\end{aligned}
$$

This holds for any values of $t_{1}, t_{2}, z$.

\subsection{A Conundrum}

Next we can consider a model in which there are still two NS fivebranes and one D fivebrane, but we consider an arbitrary number $k$ of threebranes, as in figure 10(a). To fully specify the model, we must give the linking numbers, which are $\pm\left(\frac{1}{2}-k\right)$ for the NS fivebranes, and zero for the D fivebrane. One reason to consider this model in some detail is that it is the simplest case in which we meet a certain puzzle.
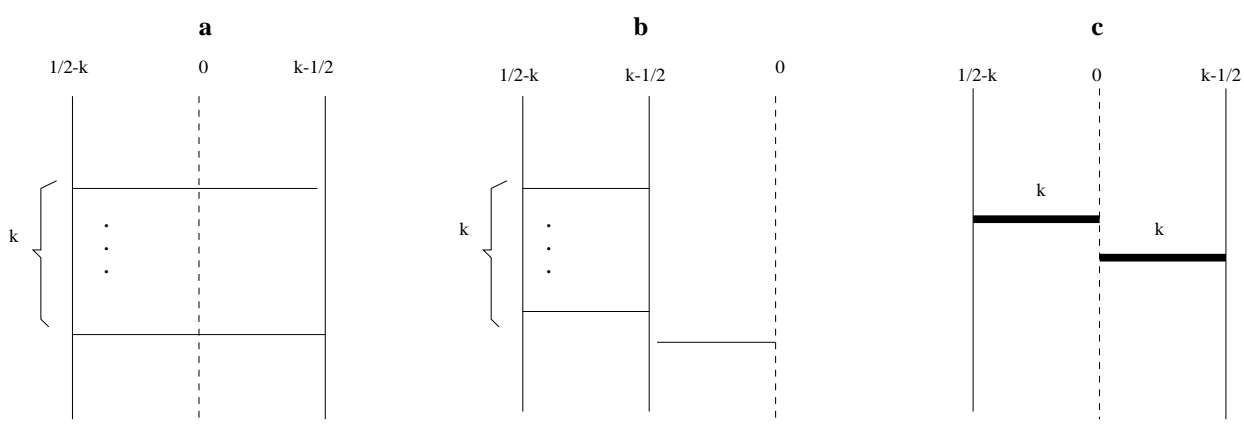

Figure 10: $U(k)$ gauge theory with one flavor and its various phases. Figure (a) and figure (b) have the same matter content and therefore are in the same phase. The vertical solid lines are solitonic fivebranes and the vertical dashed line is a fivebrane. The horizontal lines in (a) are threebranes, two of which are shown explicitly. In (c), the big fat line with a number $k$ attached to it represents $k$ threebranes which stretch between an NS fivebrane and a D fivebrane. The numbers on top of the five branes denote the linking numbers.

Applying our usual rules to figure 10(a), this theory can be identified as a $U(k)$ gauge theory with one flavor in the fundamental representation. The gauge coupling is $\left|t_{1}-t_{2}\right|$, The Fayet-Iliopoulos coupling is $\vec{w}_{1}-\vec{w}_{2}$. First we consider the case where this vanishes. The bare mass term for the hypermultiplet is $\vec{m}$, and can be set to zero by shifting the coordinates. The Colomb branch has quaternionic dimension $k$. The model has no Higgs 
branch. As in the $U(1)$ case we discussed before, the string theory description has a mysterious parameter $z$ with no obvious meaning in the low energy physics. Let us see what happens when we increase $z$ and reach the regime $t_{1}<t_{2}<z$. Again a threebrane is generated when the fivebranes cross, to give the picture of figure 10(b). Using our rules for matter fields we see that the theory remains with the same matter content. Again this flow is apparently irrelevant for this theory.

Now we try to turn on the Fayet-Iliopoulos coupling, that is to take $\vec{w}_{1}-\vec{w}_{2} \neq 0$. What happens in field theory in the present model is that this triggers supersymmetry breaking. To see this, recall that a hypermultiplet in the fundamental repreentation of $U(k)$ is equivalent from the point of view of $N=2$ supersymmetry to a pair of multiplets $A^{i}$ and $B_{j}$ in the fundamental representation and its dual (or complex conjugate). In the presence of the Fayet-Iliopoulos coupling, if the $N=2$ subalgebra is chosen suitably, the conditions for unbroken supersymmetry become

$$
\begin{aligned}
A^{i} B_{j} & =0 \\
A^{i} \bar{A}_{j}-B^{i} \bar{B}_{j} & =\delta^{i}{ }_{j} w,
\end{aligned}
$$

with $w$ the FI coupling. For $k>1$, these equations have no solution.

What happens when we embed this question in string theory, displacing the NS fivebranes to $\vec{w}_{1} \neq \vec{w}_{2}$ ? A supersymmetric configuration now cannot have a threebrane stretching between the two NS fivebranes. There are two cases to consider: (i) $t_{1}<z<t_{2}$; (ii) $t_{1}<t_{2}<z$.

In case (i), to make a supersymmetric configuration all threebranes must be broken into threebranes that go "half way," starting on the left NS fivebrane and ending on the D fivebrane, or starting on the D fivebrane and ending on the right NS fivebrane. This is sketched in figure 10(c). No moduli are possible in this picture, as threebranes starting and ending on fivebranes of opposite type are "frozen."

In case (ii), no supersymmetric configurations are possible at all. The only threebrane configuration with $t_{1}<t_{2}<z$ and linking numbers $\frac{1}{2}-k, k-\frac{1}{2}$, and 0 is the one already sketched in figure 10(b), and this is not compatible with supersymmetry when $\vec{w} \neq 0$.

Thus, in region (ii), the string theory agrees with the field theory result: supersymmetry is broken when the FI coupling is turned on. In region (i), the string theory appears to give an isolated supersymmetric vacuum, contradicting the field theory.

In general, we can see two possible resolutions of this puzzle:

(1) The parameter $z$ is not really irrelevant in the low energy $2+1$ dimensional physics. 
The classical equations (5.11) are valid when $t_{2}<z$ or $z<t_{1}$, but not when $t_{1}<z<t_{2}$, and in that latter range there is a supersymmetric vacuum in the presence of the FI terms.

(2) A configuration (like that of figure 10(c) ) with more than one threebrane connecting a given NS fivebrane to a given D fivebrane does not have a quantum state of unbroken supersymmetry, though merely drawing the picture suggests that it does.

Later, we will refer to a configuration with more than one threebrane connecting an NS fivebrane to a D fivebrane as an $s$-configuration, so the question is whether $s$-configurations can be supersymmetric.

As the above statement indicates, the physics in this model is less exotic if $s$-configurations are not supersymmetric. That is a general pattern that we will see also in other examples. Note that starting with a configuration that is not an $s$-configuration, crossing of fivebranes does not generate an $s$-configuration, so it is consistent to assume that $s$ configurations are not supersymmetric. However, we do not know a direct argument for this.

We illustrate in figure 11 some of the simplest of the bizarre possibilities that can arise if $s$-configurations are supersymmetric. In part (b), we show a $U\left(k_{1}\right)$ gauge theory coupled to an s-configuration labeled by a positive integer; by turning on an FI coupling, this can make a transition to the double $s$-configuration shown in (a).
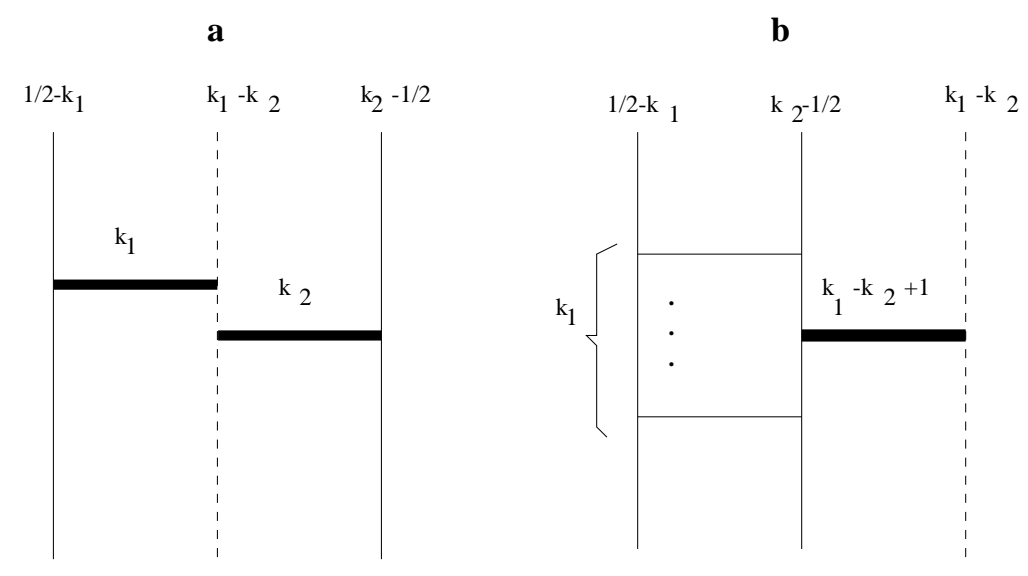

Figure 11: Exotic phases which emerge in a configuration of two solitonic five branes and a D5 brane if $s$-configurations are supersymmetric. The vertical solid lines are solitonic five branes and the vertical dashed line is a D5 brane. The horizontal lines are threebranes. The big fat line with a number $k$ attached to it represents $k$ threebranes which stretch between a D5 brane and a solitonic five brane. The numbers on top of the five branes denote the linking numbers. 


\section{Mirror Symmetry At Work}

In this section we look at the general class of theories labeled by $n_{d} \mathrm{D}$ fivebranes and $n_{s}$ NS fivebranes, with different numbers of threebranes connecting them. The number of couplings (or background fields) of the theory is $4\left(n_{d}+n_{s}\right)-7$. These are given by four transverse coordinates per brane. We are free to set the origin for all seven coordinates which are transverse to the 0,1,2 plane and this gives the above formula.

As one moves in moduli space, various phase transitions can occur:

1. Threebranes can reconnect when possible. An example for that we saw in the case of $U(1)$ with one flavor in section 5.1 .

2. Fivebranes can be rearranged, passing through each other and creating new threebranes, as in the previous section.

The only invariants under all these transitions are the "linking numbers" (integer or half integer) seen by each fivebrane. So models (but not phases) are classified by $n_{d}, n_{s}$, and the linking numbers.

Mirror symmetry is manifest in this class of models. It exchanges the $3-4-5$ directions in spacetime with the $7-8-9$, directions, so it exchanges the Fayet-Iliopolous parameters $\vec{w}$ with the bare masses $\vec{m}$. It also exchanges the two types of fivebrane, so it exchanges $n_{d}$ and $n_{s}$ while exchanging the two sets of linking numbers.

\section{1 $U(1)$ Gauge Theory With Two Flavors}

For our first experience with mirror symmetry, we consider a model with $n_{s}=n_{d}=2$, and all linking numbers zero. This model is self-mirror because $n_{s}=n_{d}$ and all linking numbers are equal.

In one obvious phase with two NS fivebranes on the outside connected by one threebrane (figure $12(\mathrm{a})$ ), this is a $U(1)$ gauge theory with two charged hypermultiplets. The self-mirror property of this model was already observed in [3], and in the present context is a consequence of the general mirror symmetry of this class of models. Mirror symmetry applied to the configuration of figure 12(a) maps it to the configuration of figure 12(b), which by going through a series of phase transitions can be mapped back to the configuration of figure 12(a). To be precise about these phase transitions, one first moves the NS fivebranes back to the outside; some new threebranes appear, as sketched in figure 12(c). Then, by reconnecting the threebranes to a single threebrane stretching between the two 

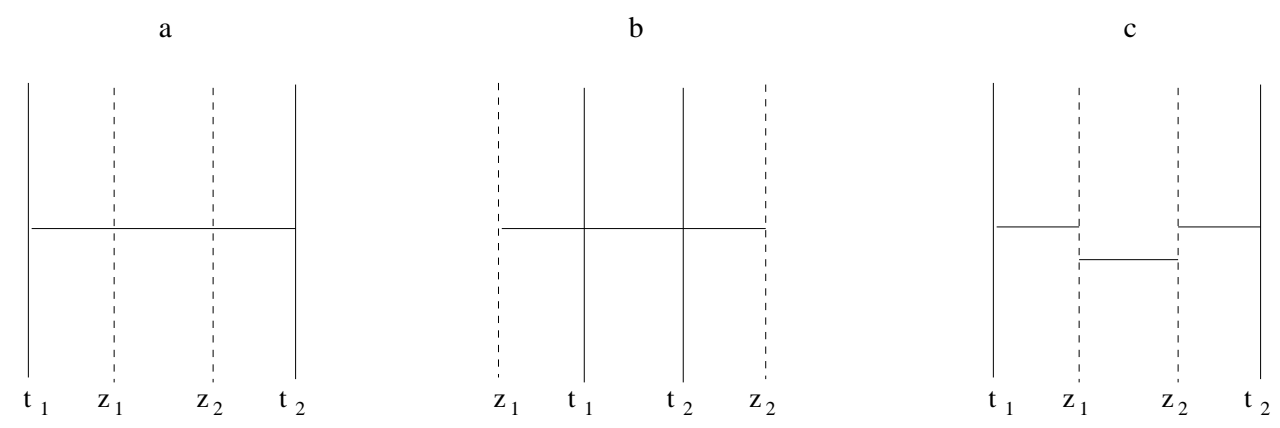

Figure 12: $U(1)$ gauge theory with two electrons.

NS fivebranes (after adjusting $\vec{w}_{1}-\vec{w}_{2}$ to zero to make this possible), one gets back to the configuration of figure 12(a). The fact that this succession of operations gets us back to the starting point is guaranteed by the fact that the linking numbers - which uniquely determine the number of threebranes between each pair of successive fivebranes - were chosen to be self-mirror.

$\mathrm{a}$

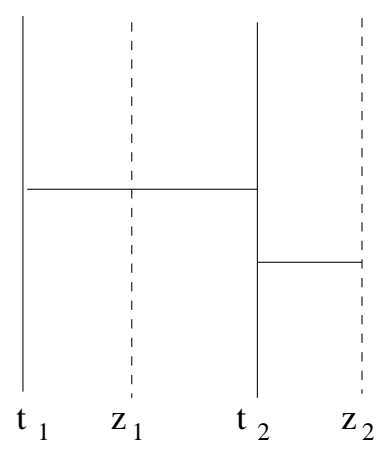

b

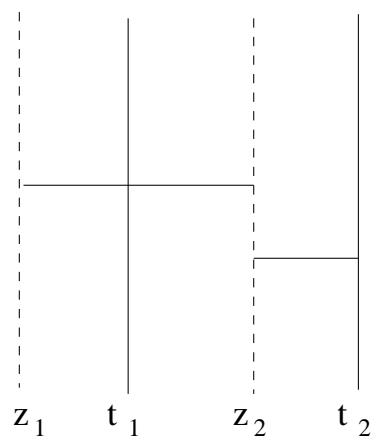

Figure 13: $U(1)$ gauge theory with two electrons for a different arrrangement of fivebranes.

It is interesting to move the fivebranes around and ask what the model looks like in other parts of the parameter space. For example, in figures 13(a,b), we consider the situation in which $t_{1}<z_{1}<t_{2}<z_{2}$. This configuration is mirror symmetric up to a rotation of, say, the $x^{1}-x^{6}$ plane that reverses the sign of $x^{6}$. (This rotation also changes the sign of $x^{1}$ and so looks like a parity transformation in the low energy $2+1$ dimensional theory.) With the threebranes connected as in figure 13(a), this looks like an electric $U(1)$ gauge theory with two charged hypermultiplets; with the threebranes connected as in figure 13(b), it looks like a magnetic $U(1)$ gauge theory with two charged hypermultiplets. The transition between them is possible only when $\vec{w}_{1}=\vec{w}_{2}$ and $\vec{m}_{1}=\vec{m}_{2}$, just as in [3].

Starting with the configuration considered in the last paragraph, it is interesting to 

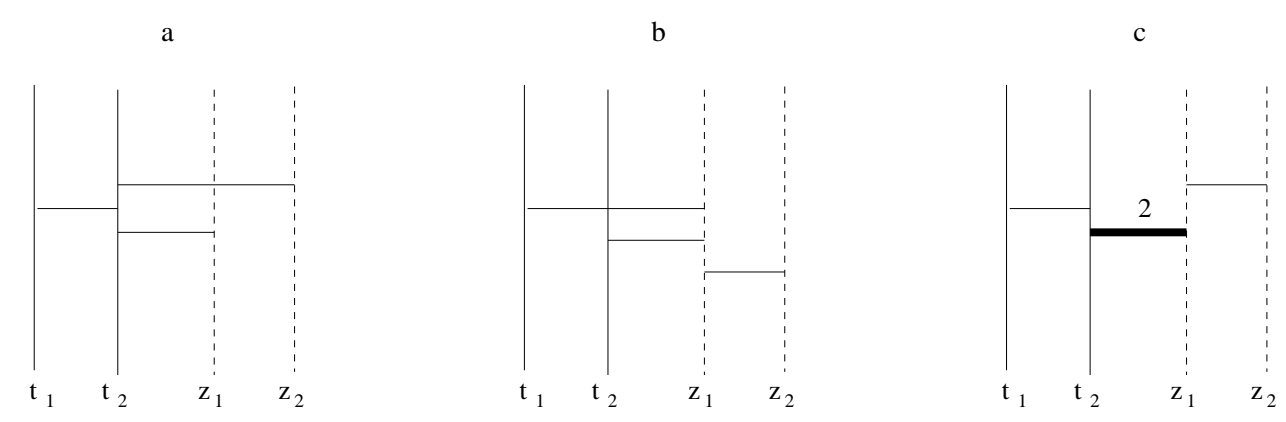

Figure 14: $U(1)$ gauge theory with two electrons in the range $t_{1}<t_{2}<z_{1}<z_{2}$. Sketched are the Coulomb branch, the Higgs branch, and an exotic branch (with two threebranes connecting the adjacent NS and D fivebranes) that exists if $s$-configurations are supersymmetric.

increase $z_{1}$ until $t_{2}<z_{1}$ and both NS fivebranes are to the left of both D fivebranes. A supersymmetric configuration in this situation is sketched in figure 14. There is both a Coulomb branch and a Higgs branch, as indicated in the figure. Applying the standard rules shows on these branches the expected spectra: $U(1)$ with two charged hypermultiplets. Also shown in the figure is a more exotic situation, with both a Higgs and a Coulomb modulus, that is possible if $s$-configurations are allowed.

In all these figures, an overall motion of D fivebranes relative to NS fivebranes is irrelevant for the $2+1$-dimensional physics unless $s$-configurations are supersymmetric. If $s$-configurations are supersymmetric, the phase in figure 14(c) is one that exists only for the given ordering of branes, so its existence can be affected by adding a constant to $z_{1}$ and $z_{2}$. This illustrates the general fact that one obtains simple and consistent results if one assumes that $s$-configurations are not supersymmetric.

Now let us go back to the most tame situation of figure 12(a). We interpret this as an electric $U(1)$ gauge theory with two charged hypermultiplets whose bare mass parameters (being controlled by the $\vec{m}$ 's of the D fivebranes) are arbitrary and in particular can vanish. When they vanish, a field theorist expects to see an enhanced $S U(2)_{d}$ global symmetry of the two hypermultiplets. To get this in our setup, we simply take the $\mathrm{D}$ fivebranes to be coincident, that is we take $z_{1}=z_{2}$. Then on the worldvolume of the $\mathrm{D}$ fivebranes there is an enhanced $U(2)$ gauge symmetry, via the Chan-Paton factors, and after factoring out the center of $U(2)$, which acts trivially on the threebranes, this gives an $S U(2)$ global symmetry in the $2+1$-dimensional low energy theory.

On the other hand, if we would think in terms of the magnetic gauge coupling, which is given by $g_{m}^{-2}=\left|z_{1}-z_{2}\right|$, it is clear that this method of obtaining an enhanced $S U(2)_{d}$ global 
symmetry involves setting $g_{m}=\infty$. Conversely, with the electric gauge coupling $g_{e}$ such that coupling $g_{e}^{-2}=\left|t_{1}-t_{2}\right|$, we will observe an enhanced $S U(2)_{n s}$ global symmetry exactly when $g_{e}=\infty$. Comparing this to [3], those authors discovered an enhanced $S U(2)_{n s}$ global symmetry precisely when $g_{e}=\infty$. In the conventional Lagrangian description, there is no parameter analogous to $g_{m}$, and (bare masses being zero) the $S U(2)_{d}$ symmetry is always present (this is the usual flavor symmetry of two massless quarks). Clearly, a major difference between the brane configurations we are considering here and what one can see in low energy field theory is that in the present description the parameters $g_{e}$ and $g_{m}$ are simultaneously visible, though their interpretations as gauge couplings hold in only part of the parameter space.

Since in known Lagrangian field theory, one is limited to $g_{m}=\infty$, mirror symmetry was seen in [3] by setting also $g_{e}=\infty$. In the present context, we see mirror symmetry for finite $g_{e}$ and $g_{m}$. The critical point of [3] with $g_{e}=g_{m}=\infty$ and $S U(2)_{n s} \times S U(2)_{d}$ symmetry corresponds to the maximally symmetric situation with all four fivebranes at the same value of $x^{6}$. This configuration can be perturbed to a nine parameter family, the parameters being three relative $x^{6}$ values, three components of $\vec{w}_{1}-\vec{w}_{2}$, and three components of $\vec{m}_{1}-\vec{m}_{2}$. (Assuming $s$-configurations are not supersymmetric, one of the nine parameters, namely an overall shift in the $z_{j}$ for fixed $t_{i}$, is generally hard to see in the $2+1$-dimensional world; the other eight parameters form two supermultiplets.) In any one of the Lagrangian realizations, say via the electric gauge group, seven of the nine parameters are visible $\left(t_{1}-t_{2}, \vec{w}_{1}-\vec{w}_{2}\right.$, and $\left.\vec{m}_{1}-\vec{m}_{2}\right)$. Some subloci of the nine parameter family are particularly difficult to understand in the low energy field theory, namely those in which a D and an NS fivebrane are still coincident.

Much of the physics of this model is controlled by the three relative distances along the $x^{6}$ direction. We have the distance between the two solitonic five branes $\left|t_{1}-t_{2}\right|$, which is an electric gauge coupling, the distance between the two D5 branes $\left|z_{1}-z_{2}\right|$, which is a magnetic gauge coupling and the distance between one solitonic five brane and one D5 brane $\left|t_{1}-z_{2}\right|$, which is more difficult to interpret. When the first distance vanishes we get the usual non-linear sigma model associated to hypermultiplet moduli spaces or instanton moduli spaces. For this particular case the relevant moduli space is that of $S U(2)$ instantons. When the second distance vanishes we get a flow of the gauge theory to a strongly coupled theory. When the third distance vanishes we get a model that must have peculiar properties, since at the "bicritical point" $z_{1}=t_{1}, z_{2}=t_{2}$, one has a model that can be perturbed to either an electric or magnetic gauge theory.

\section{Weak Coupling And Four Dimensions}


One of the most unusual features of our description, relative to conventional threedimensional field theory, is that it is possible to see at the same time electric and magnetic gauge couplings.

This is related to the fact that our threebrane world-volumes are really four-dimensional, if one looks at them closely. For the long wavelength behavior, one always has an effective three-dimensional theory, but modes that carry momentum in the fourth dimension must be included if one wants to discuss the physics at energies of order $g^{2}$. The theories we are considering here (1) have the same infrared behavior as the conventional three-dimensional gauge theories, and (2) have better duality properties, in the sense that various statements that in field theory are only true in the infrared are here exact.

If one goes to the weak coupling limit for either electric or magnetic couplings, two fivebranes become far apart, and the threebranes connecting them become macroscopically four-dimensional. An attempt to take a weak coupling limit would restore the full fourdimensional symmetry, giving a continuous mass spectrum from a three-dimensional point of view. The relevant four-dimensional theory has the full $\mathcal{N}=4$ supersymmetry, broken down to $\mathcal{N}=2$ by boundary conditions at the ends of threebranes.

\section{The Moduli Space Of Vacua}

By the Coulomb branch of the moduli space of vacua of this theory, we mean a branch that is parametrized by the position of a threebrane that ends on NS fivebranes (plus the superpartner of those variables). This is a four-dimensional hyper-Kahler manifold that exists only when $\vec{w}_{1}-\vec{w}_{2}=0$. By the Higgs branch of moduli space, we mean a branch that is parametrized by the position of a threebrane that ends on D fivebranes (plus superpartner). This is a four-dimensional hyper-Kahler manifold that exists only when $\vec{m}_{1}-\vec{m}_{2}=0$.

When $\vec{w}_{1}-\vec{w}_{2} \neq 0$ and $\vec{m}_{1}-\vec{m}_{2} \neq 0$, there is neither a Coulomb branch nor a Higgs branch. In field theory, in this situation, a standard analysis shows that there are two isolated vacua, each with a mass gap. This result is reproduced in the present context via the pictures sketched in figure 15 for the case $t_{1}<z_{1}<z_{2}<t_{2}$. Similar pictures can be drawn for other arrangements of fivebranes.

Now we will discuss the metric on the moduli space of vacua.

By looking at the system from a solitonic fivebrane point of view we identify the Coulomb branch with the moduli space of an $S U(2)$ monopole in the presence of two fixed monopoles represented by the mass deformation. The overall center of mass of the two fixed monopoles can be absorbed in a redefinition of the moduli $\vec{x}$. The metric on 
a

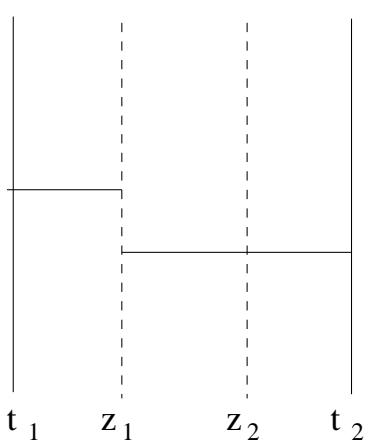

b

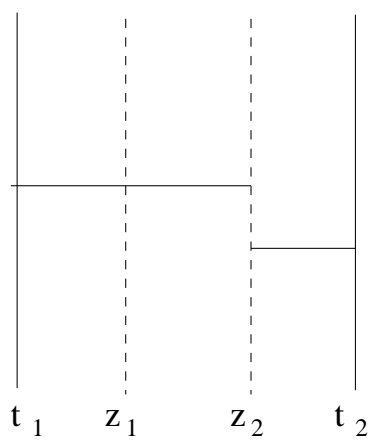

Figure 15: The two possible vacua for $U(1)$ gauge theory with two electrons with both bare mass and FI terms present.

the Coulomb branch of the moduli space has the form

$$
\begin{aligned}
d s^{2} & =G(\vec{x}) d \vec{x} \cdot d \vec{x}+G(\vec{x})^{-1} d \tilde{\theta} d \tilde{\theta}, \quad d \tilde{\theta}=d \theta-\vec{\omega} \cdot d \vec{x}, \\
G(\vec{x}) & =\left|t_{1}-t_{2}\right|+\frac{1}{|\vec{x}+\vec{m}|}+\frac{1}{|\vec{x}-\vec{m}|}, \quad \nabla_{a} \times \vec{\omega}=\nabla_{a}\left(\frac{1}{|\vec{x}|}\right) .
\end{aligned}
$$

As for the metric on the Higgs branch, at $g_{m}=\infty$ it can be determined by a classical calculation in the electric gauge theory, and is equal to the flat metric on $\mathbf{R}^{4} / \mathbf{Z}_{2}$ (which is also an $S U(2)$ instanton moduli space on $\mathbf{R}^{4}$ ). But in general we have $g_{m} \neq \infty$; the metric on the Higgs branch is naturally described via the magnetic gauge theory and is just isomorphic to what was written above:

$$
\begin{aligned}
d s^{2} & =G(\vec{y}) d \vec{y} \cdot d \vec{y}+G(\vec{y})^{-1} d \tilde{\theta} d \tilde{\theta}, \quad d \tilde{\theta}=d \theta-\vec{\omega} \cdot d \vec{y}, \\
G(\vec{x}) & =\left|z_{1}-z_{2}\right|+\frac{1}{|\vec{y}+\vec{w}|}+\frac{1}{|\vec{y}-\vec{w}|}, \quad \nabla_{b} \times \vec{\omega}=\nabla_{b}\left(\frac{1}{|\vec{y}|}\right) .
\end{aligned}
$$

This reduces to the flat metric on $\mathbf{R}^{4} / \mathbf{Z}_{2}$ when $z_{1}-z_{2}=0$ and $\vec{w}=0$.

The Fayet-Iliopoulus term can be written in the following form. Denote the two hypermultiplets by $M_{i}, \tilde{M}_{i}, i=1,2$. These fields are $N=1 d=4$ chiral multiplets. The scalars in each of these chiral multiplets transform as $(2,1)$ of $S O(3)_{H} \times S O(3)_{V}$. We collect them into a matrix form

$$
M^{A A^{\prime}}=\left(\begin{array}{cc}
M & \tilde{M}^{*} \\
-\tilde{M} & M^{*}
\end{array}\right)
$$

which satisfy the reality condition

$$
M_{A A^{\prime}}^{*}=\epsilon_{A B} \epsilon_{A^{\prime} B^{\prime}} M^{B B^{\prime}}
$$


Then the condition for a supersymmetric vacuum is

$$
\vec{w}=\frac{1}{2} \sum_{i}\left(M_{i}^{*}\right)_{A A^{\prime}} \vec{\sigma}_{B}^{A^{\prime}} M_{i}^{B A}
$$

Where $\vec{\sigma}$ are Pauli matrices. We see that when $\vec{m}=\vec{w}=0$ the global $S U(2)_{d} \times S U(2)_{H}$ (the factors are respectively the global symmetry present for coincident $D$ fivebranes and the $R$ symmetry) is broken spontanously on the Higgs branch to a diagonal subgroup. In addition the scalars $z_{i}$ couple to the gauge field through the coupling

$$
\int d^{3} x\left(A_{\mu}^{i}-\partial_{\mu} z_{i}\right)^{2}
$$

Mirror symmetry exchanges the two branches of the moduli space, the D fivebrane position $\vec{m}$ with the solitonic fivebrane position $\vec{w}$ and the gauge coupling $t_{i}$ with the hidden gauge coupling for the Higgs branch $z_{i}$. The magnetic hypermultiplets $W_{i}, \tilde{W}_{i}$, $i=1,2$ of the mirror theory can be treated similarly. Each such multiplet transforms under $(1,2)$ of $S O(3)_{H} \times S O(3)_{V}$. We collect them into a matrix form

$$
W^{A A^{\prime}}=\left(\begin{array}{cc}
W & \tilde{W}^{*} \\
-\tilde{W} & W^{*}
\end{array}\right)
$$

which satisfy the reality condition

$$
W_{A A^{\prime}}^{*}=\epsilon_{A B} \epsilon_{A^{\prime} B^{\prime}} W^{B B^{\prime}}
$$

Then the condition for a supersymmetric vacuum is

$$
\vec{m}=\frac{1}{2} \sum_{i}\left(W_{i}^{*}\right)_{A A^{\prime}} \vec{\sigma}_{B}^{A^{\prime}} W_{i}^{B A}
$$

We see that when $\vec{m}=\vec{w}=0$ the global $S U(2)_{s} \times S U(2)_{V}$ is broken spontanously on the Coulomb branch to a diagonal subgroup. In addition the scalars $t_{i}$ couple to the gauge field through the coupling

$$
\int d^{3} x\left(B_{\mu}^{i}-\partial_{\mu} t_{i}\right)^{2}
$$

\section{Higher Rank Gauge Groups}

Now we will briefly consider a larger class of models still with two NS fivebranes and two D fivebranes but with more general linking numbers. We start with a NS-D-D-NS configuration and assign linking numbers $k_{1}, k_{2}, k_{3}, k_{4}$ to the five branes. These numbers are invariants of the theories. We denote by $n_{1}, n_{2}, n_{3}$ the number of D3 branes between 
each two adjacent five branes starting from the left. Using equations 5.10 and 5.9 they are related to $k$ 's by the formulas

$$
k_{1}=1-n_{1}, \quad k_{2}=n_{1}-n_{2}, \quad k_{3}=n_{2}-n_{3}, \quad k_{4}=n_{3}-1 .
$$

Note that the linking numbers sum to zero, reflecting the fact that there are no sources for linking number at the boundary. As we reorder the fivebranes, the number of threebranes will change in such a way that the linking numbers remain invariant. Once we know these numbers we can use our rules in section 3 to find the spectrum in any particular phase. The different phases and corresponding numbers are given in the table below. The threebrane numbers do not completely specify a branch of vacua, since one must also specify how the threebranes are connected, as we discussed in some detail above for $k_{1}=\ldots=k_{4}=0$.

\begin{tabular}{|c|c|c|c||c|c|c|}
\hline $\mathrm{NS}$ & $\mathrm{D}$ & $\mathrm{D}$ & $\mathrm{NS}$ & $n_{1}$ & $n_{2}$ & $n_{3}$ \\
\hline $\mathrm{NS}$ & $\mathrm{D}$ & $\mathrm{NS}$ & $\mathrm{D}$ & $n_{1}$ & $n_{2}$ & $n_{2}-n_{3}+1$ \\
\hline $\mathrm{NS}$ & $\mathrm{NS}$ & $\mathrm{D}$ & $\mathrm{D}$ & $n_{1}$ & $n_{1}-n_{3}+2$ & $n_{2}-n_{3}+1$ \\
\hline $\mathrm{D}$ & $\mathrm{NS}$ & $\mathrm{NS}$ & $\mathrm{D}$ & $n_{2}-n_{1}+1$ & $n_{2}$ & $n_{2}-n_{3}+1$ \\
\hline
\end{tabular}

Some additional orderings are related to these by obvious symmetries. Only orderings in which the threebrane numbers are all non-negative can give supersymmmetric vacua.

In most cases, for most values of the $n_{i}$, to analyze this model one meets our basic question of whether $s$-configurations are supersymmetric.

\subsection{Some Further Models}

Now we will analyze in some detail a model that is not self-mirror, but has a known mirror. This is the model with $n_{s}=2 \mathrm{NS}$ fivebranes and $n_{d}=m \mathrm{D}$ fivebrames. It is mirror to the case of $m$ NS and $2 \mathrm{D}$ fivebranes and gives, as we will see, one of the examples of Intriligator and Seiberg [3].

First we consider, as in figure 16 the case that $t_{1}<z_{1}<\cdots,<z_{m}<t_{2}$. We take the linking numbers to be zero for the $\mathrm{D}$ fivebranes and $m / 2-1$ for the left solitonic fivebrane and $1-m / 2$ for the right one. For $\vec{w}_{1}=\vec{w}_{2}$, there is a phase with one threebrane which stretches between the two solitonic five branes. In this phase, we can identify the theory as a $U(1)$ gauge theory with $m$ charged hypermultiplets.

The Coulomb branch is four-dimensional and is parametrized as usual by the scalars (the position $\vec{x}$ and the scalar dual to the photon) associated with the threebrane. The 


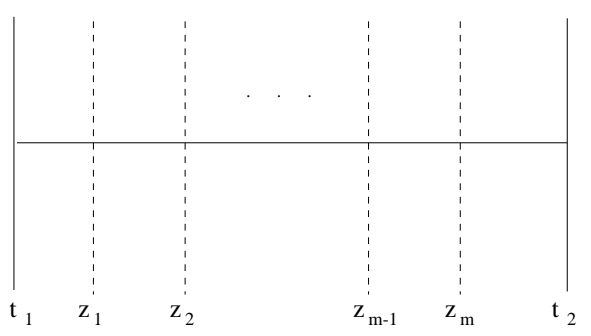

b

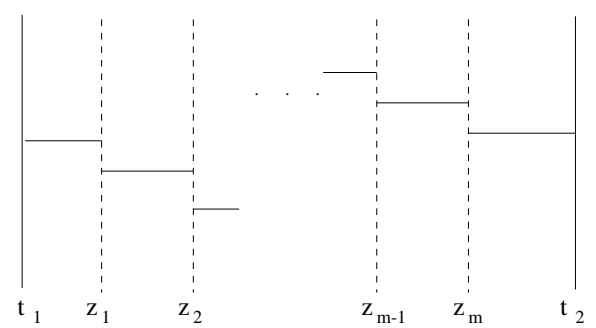

Figure 16: $U(1)$ gauge theory with $m$ electrons.

hypermultiplet bare masses are given by the positions $\vec{m}_{i}$ of the $\mathrm{D}$ fivebranes. The center of mass of the $\mathrm{D}$ fivebranes can be absorbed into a redefinition of the threebrane position and thus we have $m-1$ independent mass parameters. The difference $\vec{w}_{1}-\vec{w}_{2}$ is a Fayet-Iliopoulos coupling; the Coulomb branch only exists if it vanishes.

With the same values of the couplings ( such as $t_{i}$ and $z_{j}$ ), there is also a Higgs branch in which the threebranes are connected differently. This is sketched in figure 16(b). The Higgs branch is parametrized by $m-1$ threebranes which stretch between two adjacent D fivebranes.

a

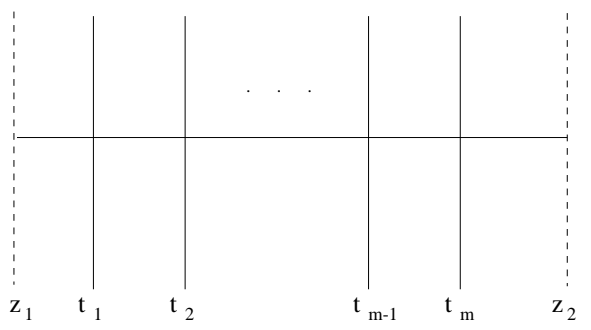

b

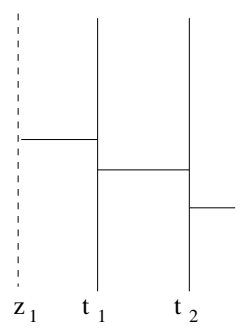

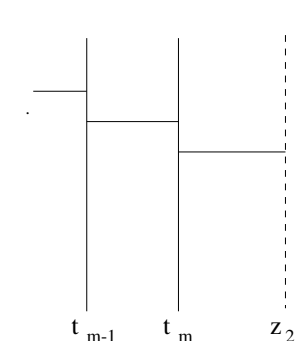

Figure 17: $U(1)$ gauge theory with $m$ electrons in the mirror description.

Now we apply mirror symmetry to the Higgs branch, which turns into the configuration of figure 17 with two D fivebranes on the outside and $m$ NS fivebranes on the inside. In this case, we see a $U(1)^{m-1}$ gauge theory, one $U(1)$ factor for each threebrane connecting two adjacent NS fivebranes. Whenever two consecutive threebranes meet in space one gets a massless hypermultiplet, so this theory has $m$ hypermultiplets, of charges $(-1,0, \ldots, 0)$, $(1,-1,0, \ldots, 0),(0,1,-1,0, \ldots, 0), \cdots,(0, \ldots, 0,1,-1),(0, \ldots, 0,1)$. So the $U(1)$ theory with $m$ hypermultiplets must be mirror to the $U(1)^{m-1}$ theory with that particular spectrum. This is a result of Intriligator and Seiberg [3]. As in their description, there is an $S U(m)$ global symmetry when the $m$ D fivebranes, in the original description, are coincident, and an $S U(2)$ global symmetry when the electric gauge coupling of the original 
description is infinite, that is when $t_{1}=t_{2}$.

In our discussion of the Higgs branch, we looked at the maximum dimension component with the largest number of threebranes, with threebranes connecting consecutive fivebranes. It is possible to consider other cases. In the description in terms of the magnetic gauge group $U(1)^{m-1}$, these can be interpreted in terms of partial Higgsing.

\section{$U(2)$ With $m$ Flavors}

Next we consider a $U(2)$ gauge theory coupled to $m$ flavors. This can be realized by the brane configuration of figure 18 .

a

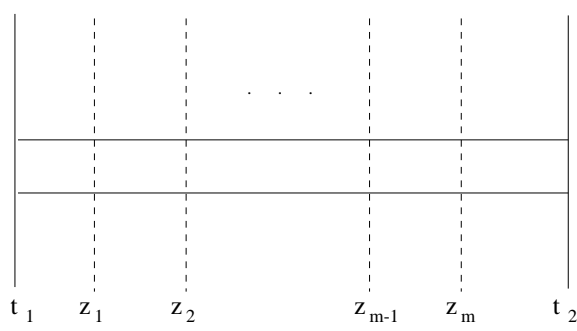

$\mathrm{b}$

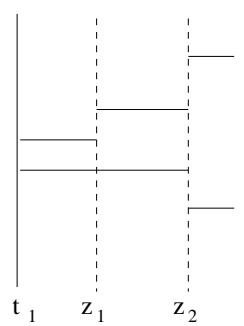

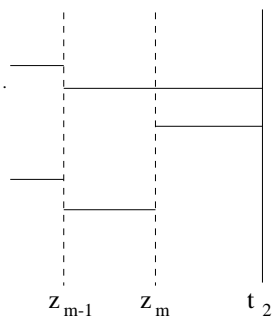

Figure 18: $U(2)$ gauge theory with $m$ flavors. The Coulomb and Higgs branches are sketched, respectively, in (a) and (b).

If we henceforth count dimensions in the quaternionic sense, then the Coulomb branch is two-dimensional and the Higgs branch is $2 m-4$-dimensional. We tune the masses to the origin in order to allow for threebranes to be created. A Higgs phase is shown in figure 18(b). The magnetic gauge group is is $U(1) \times U(2)^{m-3} \times U(1)$ with in an obvious notation hypermultiplets in the representation $(\mathbf{1}, \mathbf{2}) \oplus \mathbf{2} \oplus(\mathbf{2}, \mathbf{2}) \oplus \ldots \oplus(\mathbf{2}, \mathbf{2}) \oplus \mathbf{2} \oplus(\mathbf{2}, \mathbf{1})$, coming from strings which stretch between $\mathrm{D}$ threebranes along $\mathrm{D}$ fivebranes. This system has a Coulomb branch of dimension $2 m-4$ and a Higgs branch of dimension 2 which gives the required dimension for the dual theory.

This is a derivation of the mirror symmetry for a slightly different model from the one found by [3]. In that example the gauge group is $S U(2)$. How to compare the two models is discussed at the end of this paper.

\subsection{More Mirrors}

In this section we will find mirrors for $U(k)$ gauge theories with $m$ flavors for all $k$ and $m$, generalizing the above. We first need to construct such a theory using branes. Using our rules from section 3 we find that a configuration of two NS fivebranes and $m$ 
$\mathrm{D}$ fivebranes provides the right setting. The five branes are ordered by $t_{1}<z_{1}<\cdots<$ $z_{n}<t_{2}$ as in figure 19 .
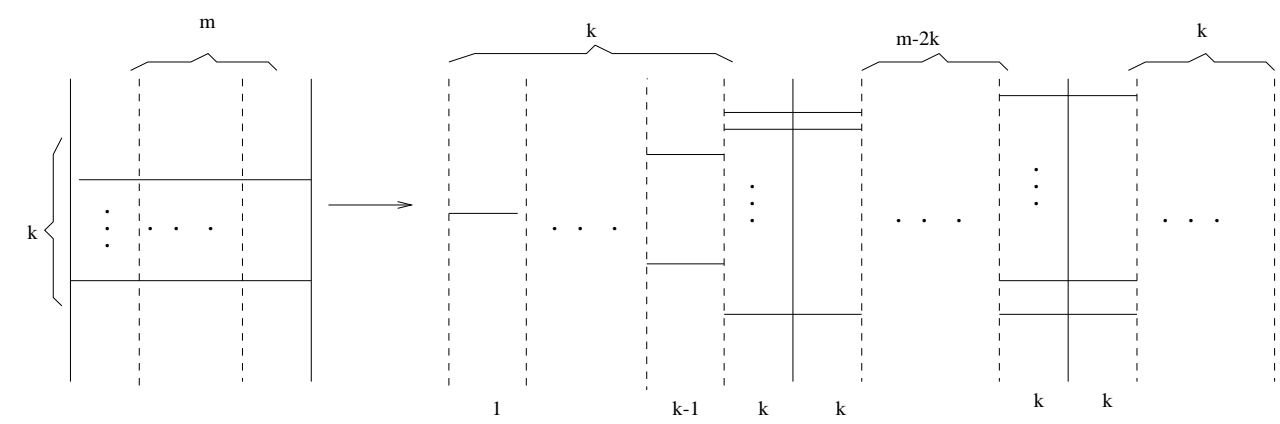

Figure 19: A fivebrane configuration with $U(k)$ gauge group coupled to $m$ flavors, and its magnetic dual. In figure (a) there are $m$ D fivebranes (dashed vertical lines) and $k$ threebranes (horizontal lines) which stretch between two solitonic fivebranes (solid vertical lines). This is the electric theory. In figure (b) we have the magnetic dual. The numbers written between a pair of adjacent fivebranes equals the number of threebranes stretched in between.

We start with an electric theory which has $k$ threebranes stretched between the two solitonic five branes. It represents a gauge group $U(k)$. The linking numbers are zero for the D5 branes, $m / 2-k$ for the left solitonic five brane and $k-m / 2$ for the right one. Note that for the special value $m=2 k$ the linking numbers for the solitonic fivebranes changes sign. This hints that the behavior of the theories for cases above and below this critical value will be different. That is actually so. In fact, if the bare masses and FI term are zero, complete Higgsing is only possible for $m \geq 2 k$. This is closely related to a result of [26], where the $S U(k)$ theory with $m$ flavors was analyzed and it was shown that for $m<2 k$, complete Higgsing occurred only on a "baryonic branch." (The classical Higgs branch is independent of the spacetime dimension in the range from three to six; the work of [26] was motivated by the four-dimensional model.) In our case, the gauge group is $U(k)$ instead of $S U(k)$; the gauging of the extra $U(1)$ kills the baryonic branch, since the "baryons" are not $U(1)$-invariant. It is presumably no coincidence that in the $d=4$ theory, the beta function of the $S U(k)$ part of the gauge group changes sign for $m=2 k$. Going back to three dimensions, the fact that complete Higgsing is only possible for $m \geq 2 k$ means that a mirror - understood as a model whose Coulomb branch is the Higgs branch of the original model - can only exist in this range of $m$.

We will study the phases of the model by the usual techniques of rearrangement of branes. This produces a rich structure of phases and provides a web of phase transitions; most of the phases (modulo possible $s$-configurations) are mixtures of Coulomb and Higgs 
branches, described in various ways. To find a mirror, we must go to a Higgs branch in which all moduli are derived from positions of threebranes that end on D fivebranes; after this, we perform a mirror transformation and reinterpret the moduli space as the Coulomb branch of a gauge theory. Though the answer we will get is complicated, it is obtained by straightforward implementation of the rules that we have described. We want to find a mirror with zero bare masses and FI terms; those can always then be added as perturbations.

There are many ways to go to a Higgs branch. One approach is to move $k \mathrm{D}$ fivebranes to the left and $k \mathrm{D}$ fivebranes to the right. Note that to do that we assume that $m \geq 2 k$. The masses and FI terms are set to zero to allow for transitions which will generate D3 branes. The fivebranes are ordered along the $x^{6}$ direction as $z_{1}<\cdots z_{k}<t_{1}<z_{k+1}<\cdots<z_{m-k}<t_{2}<z_{m-k+1}<\cdots<z_{m}$. The linking numbers are invariant under such reorderings and lead to generation of threebranes for each reordering. The linking numbers determine uniquely the number of threebranes between any two fivebranes. They are $1,2, \ldots, k, \ldots, k, k-1, \ldots, 1$. We can now connect and break the threebranes in such a way that they will be stretched between D fivebranes only. (This can be done without ever passing through an $s$-configuration as an intermediate state, so there is no need to assume they exist.) After stretching all threebranes between $\mathrm{D}$ fivebranes as in the figure, the magnetic gauge theory is $U(1) \times U(2) \times$ $\cdots \times U(k-1) \times U(k)^{m-2 k+1} \times U(k-1) \times \cdots \times U(1)$ with hypermultiplets transforming as $(\mathbf{1}, \mathbf{2}) \oplus \ldots \oplus(\mathbf{k}-\mathbf{1}, \overline{\mathbf{k}}) \oplus \mathbf{k} \oplus(\mathbf{k}, \overline{\mathbf{k}}) \oplus \ldots \oplus(\mathbf{k}, \overline{\mathbf{k}}) \oplus \mathbf{k} \oplus(\mathbf{k}, \overline{\mathbf{k}-\mathbf{1}}) \oplus \ldots \oplus(\mathbf{2}, \mathbf{1})$. The dimension of the Coulomb branch of this magnetic theory is calculated by counting the number of threebranes and is $k(m-k)$, which equals the dimension of the Higgs branch of the original electric theory; likewise, the magnetic theory has a Higgs branch of dimension $k$, the dimension of the original Coulomb branch.

One could also have gone to the Higgs branch without moving fivebranes at all, just by reconnecting threebranes. For a Higgs branch, all threebranes either (a) connect an NS fivebrane to a D fivebrane and so have no moduli, or (b) connect to D fivebranes to each other and so represent electric hypermultiplets (or magnetic vectors). The Higgs branch must then be a situation in which all $k$ threebranes emanating from a NS fivebrane end on D fivebranes. Assuming that $s$-configurations are not supersymmetric, they must end on $k$ distinct $\mathrm{D}$ fivebranes. For $m \geq 2 k$, it is possible to arrange so that each $\mathrm{D}$ fivebrane is connected by a threebrane to at most one NS fivebrane; this will then give a point on a Higgs branch. For $m<2 k$, some $\mathrm{D}$ fivebrane is connected by threebranes to both NS fivebranes. Those threebranes could reconnect to give a threebrane suspended between the two NS fivebranes, showing that the configuration in question is on a branch with at 
least one massless vector multiplet, and complete Higgsing has not occurred. In this way, we recover from the brane diagrams the field theory result that complete Higgsing does not occur for $m<2 k$.

For $m \geq 2 k$, one can go on in this way and use the brane pictures to compute the dimension of the Higgs branch. For this, it is necessary to make sure to use a brane picture which is generic (and does not represent a sublocus of smaller dimension). Generically, the $k$ threebranes connecting an NS fivebrane to D fivebranes should connnect it to the closest ones (otherwise, these threebranes could break); with this understood, the number of Higgs moduli - interpreted as the number of variable positions of threebranes that connect D fivebranes - is $(m-1) k-2(1+2+\ldots+(k-1))=k(m-k)$, in agreement with the field theory answer. The description we have given can actually be made more precise; the threebrane positions correspond to the matrix elements of the Higgs matrices used in [26].

\section{Continuation Past Infinite Coupling}

So far we have mainly considered transitions in which the ordering of two fivebranes of opposite type is changed. In such a transition, a threebrane is created but (if $s$ configurations are not supersymmetric) the field content of the low energy theory does not change. Let us now briefly consider the case of reordering two fivebranes of the same type that have different linking numbers.

In this particular example, consider moving the rightmost NS fivebrane to the extreme left and vice-versa. If one simply keeps track of the linking numbers, one sees that this process should turn a $U(k)$ theory with $m$ flavors into a $U(m-k)$ theory with $m$ flavors. This is an attractive result, very reminiscent of Seiberg duality in four dimensions. But there is obviously a problem if $m-k$ is negative.

We propose that the resolution of this is that if the relative $D$-terms vanish, then the two NS fivebranes, when they meet in $x^{6}$, actually meet in spacetime and may exchange magnetic charge. As a result, the transition proposed in the last paragraph might not occur.

To avoid magnetic charge exchange, one can first turn on a $D$ term so that the NS fivebranes can be reordered in $x^{6}$ without meeting in spacetime. We have already seen that turning on the $D$ term while preserving supersymmetry requires $m \geq k$. For this range of $m$ and $k$, by first turning on the $D$ term, then moving the NS fivebranes, and then turning the $D$ term back off, we can indeed interpolate between the $U(k)$ and $U(m-k)$ theories with $m$ flavors. 
For $m<k$, where one is restricted to $D=0$, if one tries to reorder the two NS fivebranes, magnetic charge exchange must occur when the fivebranes meet in spacetime, to avoid the contradiction of a $U(m-k)$ gauge group with $m-k<0$. For $m \geq k$, the magnetic charge exchange does not occur for non-zero $D$ term, and it is very plausible that it does not occur also when the $D$ term is zero.

If so, then the point at which the two NS fivebranes meet in spacetime is a very interesting exotic fixed point. It is a kind of common strong coupling limit of the $U(k)$ theory with $m$ flavors and the $U(m-k)$ theory with $m$ flavors. From this point of view, either one of these theories is the analytic continuation of the other to negative values of $1 / g^{2}$.

\section{Mirror For $S U(k)$}

It is also interesting to obtain a mirror for a $S U(k)$ gauge theory with $m$ flavors. This is formally obtained from the $U(k)$ theory that we have already studied by ungauging the $U(1)$ which is the center of $U(k)$. Ungauging the $U(1)$ reduces the dimension of the Coulomb branch by one and increases the dimension of the Higgs branch by one. We should look for an operation on the mirror theory that has the opposite effect: increasing by one the dimension of the Coulomb branch and reducing by one the dimension of the Higgs branch. We conjecture that the mirror of ungauging a $U(1)$ is gauging a $U(1)$, which has this effect.

Here is a heuristic reason for the conjecture to be true. Consider any gauge theory $T$ with a $U(1)$ gauge field $A$ with gauge transformation law $A_{i} \rightarrow A_{i}-\partial_{i} a, a$ being the gauge parameter. Ungauging can be accomplished by adding another hypermultiplet $H$ with a gauge transformation law $H \rightarrow H+\epsilon a$, where $\epsilon$ is an arbitrary constant. No matter how small $\epsilon$ is, $H$ can be gauged away, the $U(1)$ gets a mass and can be integrated out, and the theory $T^{\prime}$ with the $H$ field is equivalent at long distances to the $T$ theory with the $U(1)$ ungauged. Now, let us look for a mirror of $T^{\prime}$. Because $\epsilon$ can be arbitrarily small, $H$ can be treated as a spectator, and if a mirror $\tilde{T}$ is known for $T$, a mirror $\tilde{T}^{\prime}$ can be found for $T^{\prime}$ just by performing the $T \rightarrow \tilde{T}$ mirror transformation in the presence of $H$. Under this transformation, $H$, being a hypermultiplet, will very plausibly be reinterpreted as a $U(1)$ vector multiplet, and if so the $\tilde{T}^{\prime}$ theory will be $\tilde{T}$ with gauging of an extra $U(1)$. (It is interesting to note that such gauging of $U(1)$ 's that generate translations as well as rotations is important in constructions of some of the hyper-Kahler manifolds we met in section five as hyper-Kahler quotients of Euclidean space [27].)

Going back to our particular problem, it has two obvious global $U(1)$ symmetries, 
which come from the $U(1)$ gauge symmetries on the NS fivebrane worldvolumes, interpreted as global symmetries in the $2+1$-dimensional world. We want to gauge a combination of the two $U(1)$ 's, but as the diagonal $U(1)$ (which gauges overall translations) decouples anyway, there is no harm in gauging both of these $U(1)$ 's. The magnetic dual we obtained above, once one gauges both of the extra $U(1)$ 's, can be described by the quiver diagram of figure 20. In a quiver diagram, each node labeled by an integer $a$ rep-

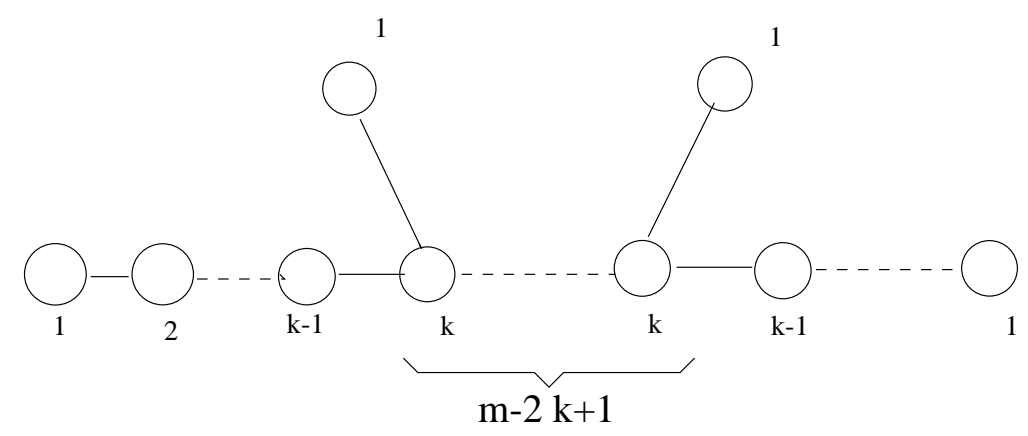

Figure 20: A Dynkin diagram which represents the magnetic theory which is dual to $S U(k)$ with $m$ flavors. The nodes represent factors of the gauge group and the lines connecting nodes represent hypermultiplets.

resents a $U(a)$ factor in the gauge group, and each line between two nodes labeled by $a, b$ represents a hypermultiplet transforming as $(\mathbf{a}, \overline{\mathbf{b}})$ under $U(a) \times U(b)$. Note that the particular quiver diagram in figure 20, in the case $k=2$, is the quiver associated with the $D_{n}$ extended Dynkin diagram. Our assertion that the theory associated with the quiver in the figure represents the mirror of the $S U(k)$ theory with $m$ hypermultiplets reduces for $k=2$ to an assertion by Intriligator and Seiberg [3].

\section{Acknowledgements}

We would like to acknowledge helpful discussions with G. Chalmers, O. Ganor, V. Sadov, N. Seiberg, and M. Strassler. While this work was nearing completion two additional papers explaining three-dimensional mirror symmetry in some situations appeared $[28,29]$.

\section{References}

[1] N. Seiberg and E. Witten, "Gauge dynamics and compactification to three dimensions.," hep-th/9607163. 
[2] G. Chalmers and A. Hanany, "Three dimensional gauge theories and monopoles," hep-th/9608105.

[3] K. Intriligator and N. Seiberg, "Mirror symmetry in three dimensional gauge theories.," Phys. Lett. B387 (1996) 513-519, hep-th/9607207.

[4] C. G. Callan, J. A. Harvey, and A. Strominger, "Supersymmetric string solitons," hep-th/9112030.

[5] C. G. Callan, J. A. Harvey, and A. Strominger, "World sheet approach to heterotic instantons and solitons," Nucl. Phys. B359 (1991) 611.

[6] C. G. Callan, J. A. Harvey, and A. Strominger, "Worldbrane actions for string solitons," Nucl. Phys. B367 (1991) 60-82.

[7] A. Strominger, "Open p-branes," Phys. Lett. B383 (1996) 44-47, hep-th/9512059.

[8] P. K. Townsend, "D-branes from m-branes," Phys. Lett. B373 (1996) 68-75, hep-th/9512062.

[9] T. Banks, M. R. Douglas, and N. Seiberg, "Probing F-theory with branes.," Phys. Lett. B387 (1996) 278-281, hep-th/9605199.

[10] N. Seiberg, "IR dynamics on branes and space-time geometry.," Phys. Lett. B384 (1996) 81-85, hep-th/9606017.

[11] A. A. Tseytlin, "Self-duality of Born-Infeld action and Dirichlet 3-brane of type IIB superstring theory," Nucl. Phys. B469 (1996) 51-67, hep-th/9602064.

[12] M. B. Green and M. Gutperle, "Comments on three-branes," Phys. Lett. B377 (1996) 28-35, hep-th/9602077.

[13] M. F. Atiyah and N. J. Hitchin, "The Geometry and Dynamics of Magnetic Monopoles," in M.B. Porter Lectures, p. 133p, Princeton, USA: univ. pr., 1988.

[14] D.-E. Diaconescu, "D-branes, monopoles and Nahm equations," hep-th/9608163.

[15] E. J. Weinberg, "Fundamental monopoles and multi - monopole solutions for arbitrary simple gauge groups," Nucl. Phys. B167 (1980) 500.

[16] M. K. Murray, "A note on the $(1,1, \ldots, 1)$ monopole metric," hep-th/9605054.

[17] P. Kronheimer MS. thesis, Oxford University, unpublished (1985). 
[18] S. A. Connell, "The dynamics of the $S U(3)(1,1)$ magnetic monopole," unpublished (1991) ftp://maths.adelaide.edu.au/pure/mmurray/oneone.tex.

[19] J. P. Gauntlett and D. A. Lowe, "Dyons and S-duality in N=4 supersymmetric gauge theory," Nucl. Phys. B472 (1996) 194-206, hep-th/9601085.

[20] K. Lee, E. J. Weinberg, and P. Yi, "Electromagnetic duality and $S U(3)$ monopoles," Phys. Lett. B376 (1996) 97-102, hep-th/9601097.

[21] U. Lindstrom and M. Rocek, "Scalar tensor duality and $\mathrm{N}=1,2$ nonlinear sigma models," Nucl. Phys. B222 (1983) 285.

[22] H. Pedersen and S. P. Yat, "Hyperkahler metrics and a generalization of the bogomolny equations," Commun. Math. Phys. 117 (1988) 569.

[23] K. Lee, E. J. Weinberg, and P. Yi, "The moduli space of many BPS monopoles for arbitrary gauge groups," Phys. Rev. D54 (1996) 1633-1643, hep-th/9602167.

[24] G. Chalmers, "Multi-monopole moduli spaces for SU(N) gauge group.," hep-th/9605182.

[25] G. W. Gibbons, "The Sen conjecture for fundamental monopoles of distinct type," Phys. Lett. B382 (1996) 53-59, hep-th/9603176.

[26] P. C. Argyres, M. R. Plesser, and N. Seiberg, "The moduli space of N=2 susy QCD and duality in N=1 susy QCD," Nucl. Phys. B471 (1996) 159-194, hep-th/9603042.

[27] G. W. Gibbons and P. Rychenkova, "Hyperkaehler quotient construction of BPS monopole moduli spaces.," hep-th/9608085.

[28] J. de Boer, K. Hori, H. Ooguri, and Y. Oz, "Mirror symmetry in three-dimensional gauge theories, quivers and d-branes," hep-th/9611063.

[29] M. Porrati and A. Zaffaroni, "M-theory origin of mirror symmetry in three dimensional gauge theories," hep-th/9611201. 